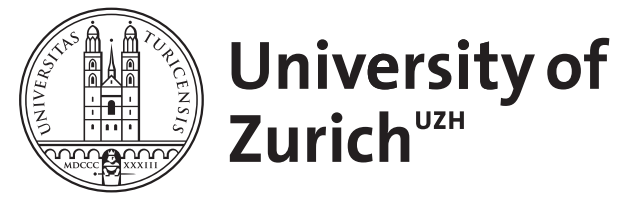
Archive

University of Zurich

University Library

Strickhofstrasse 39

CH-8057 Zurich

www.zora.uzh.ch

Year: 1988

\title{
Equilibrium configurations of crystals
}

Chipot, M ; Kinderlehrer, D

DOI: https://doi.org/10.1007/BF00251759

Posted at the Zurich Open Repository and Archive, University of Zurich

ZORA URL: https://doi.org/10.5167/uzh-22951

Journal Article

Originally published at:

Chipot, M; Kinderlehrer, D (1988). Equilibrium configurations of crystals. Archiv for Rational Mechanics and Analysis, 103(3):237-277.

DOI: https://doi.org/10.1007/BF00251759 
EQUILIBRIUM CONFIGURATIONS OF CRYSTALS

BY

MicheL CHIPOT

AND

DAVID KinDERLEHRER

IMA Preprint Series \# 326

May 1987

\section{INSTITUTE FOR MATHEMATICS AND ITS APPLICATIONS}

UNIVERSITY OF MINNESOTA

514 Vincent Hall

206 Church Street S.E.

Minneadolls. Minnesota 55455 


\section{Equilibrium configurations of crystals}

Michel Chipot and David Kinderlehrer

\section{Contents}

1. Introduction

2. A useful construction

3. Subenergy and homogeneous deformation

4. General boundary conditions and the existence of parametrized measures

5. Parametrized measure minima

6. Stable parametrized measure minima

7. Parametrized measure equilibria

8. Some concluding remarks

\section{Introduction}

The morphology of a crystal may show several phases and these may be altered with changes in its mechanical or thermal environment. For example some crystals may be deformed to consist of several twin related phases. To understand this in the context of thermoelasticity, Ericksen [21-23],[25-31] has derived a stored energy density which shows invariance with respect to change of the crystallographic lattice basis of the material. Such a density is invariant with respect to an infinite discrete group as well as frame indifferent. A body governed by it is rendered highly unstable with respect to certain motions. For example, at a smooth local minimum of energy in a constant temperature heat bath, the Cauchy stress reduces to a pressure, cf. Ericksen [20]. So it seems unlikely that even setting homogeneous boundary conditions leads to a homogeneous extremal.

In this note we wish to determine by direct methods equilibrium configurations under displacement loading conditions. We favor this approach as a means of surmounting the difficulties imposed by the defect structures on the stability of smooth solutions. Our first objective, then, is to calculate the minimum energy of a configuration. An important role in the thermodynamics of the crystal is played by its subenergy, introduced by Ericksen [24] and based in part on a method of Flory [32]. In many 


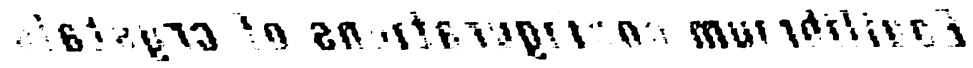

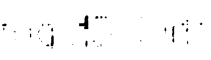

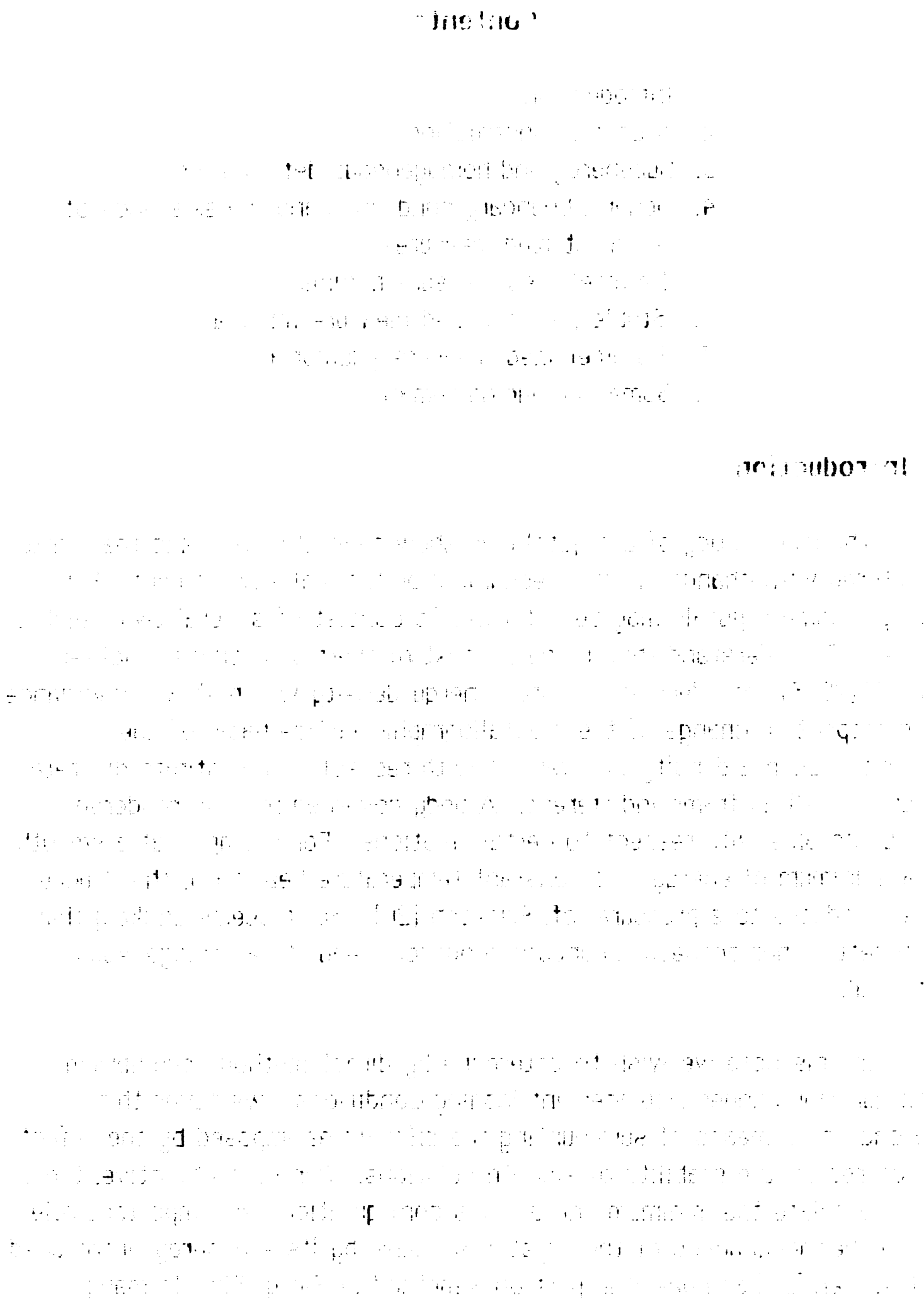


cases, the energy assumed by a configuration is its subenergy and its stress is a pressure determined by it. This may be reconciled with one common thermodynamic view where phase diagrams are expressed in terms of pressure, or specific volume, and temperature [66]. What this means from the perspective of analysis and why it should be so we may succeed in clarifying in the course of this discussion.

There are two facets to these questions as topics in the calculus of variations. One is to study energy functions in general to seek those properties of material symmetry and continuity which permit resolution of minimum energy issues. Fonseca $[34,35]$ has undertaken an analysis in this direction. Another is to restrict attention to the elastic crystal to assess the behavior of minimizing sequences and their various state functions. This is the topic here. The two methods are complementary and they agree in their calculation of the minimum energy available to a configuration.

A viewpoint that we shall adopt is that one reasonable choice of solutions is given by measure valued solutions, or parametrized measures in the sense of Young [78]. These have been introduced to the study of differential equations by Tartar [75] in order to study hyperbolic conservation laws, $\mathrm{cf}$. also $[16,73]$. They are reasonable in the sense that they predict state functions of the deformed body. For instance, the energy and the stress may be calculated. Morphology may be ascertained by inspection of the linearized equation that the parametrized measure provides. The parametrized measure serves as an accounting device to summarize properties of a minimizing sequence.

What emerges of these considerations is a coarse theory which in some manner accomodates information from a finer structure to yield macroscopic information. A limit configuration found in this way may be a macroscopically homogeneous but infinitely twinned array of states of minimum energy. It would seem that the crystal seeks to assume the lowest energy available to it by suffering small, kinematically admissible shears. Of course, a physical crystal cannot be infinitely twinned, but what we have constructed here can offer an approximation to the actual deformed body. From this point of view, the parametrized measure records mechanical properties of the microstructure.

At the conclusion of this paper we attempt to elaborate on these ideas and to provide the reader with some indication of the presence of fine scale structures, or microstructures, in nature. 
KEO , OS VAM

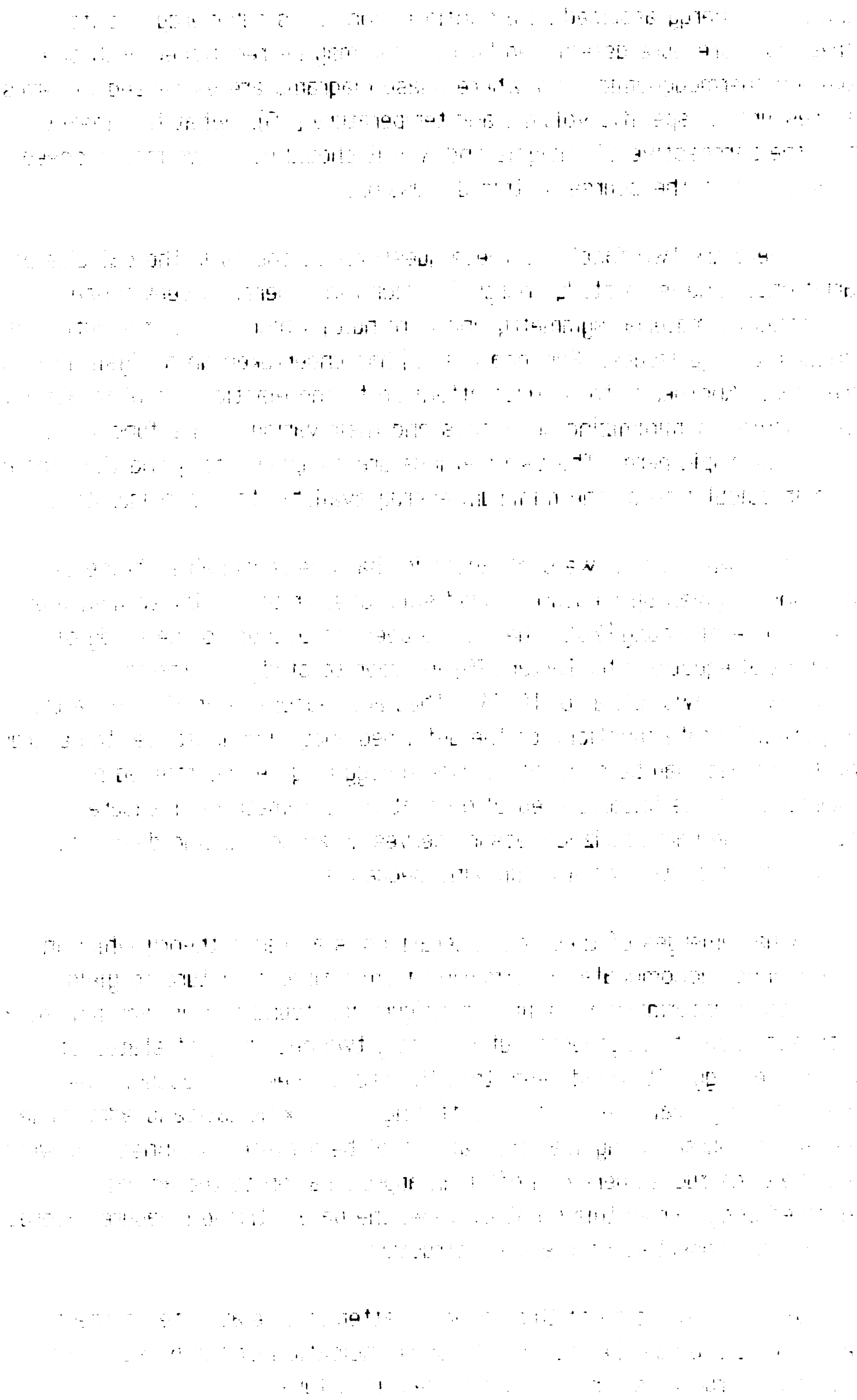


Ericksen's ideas have stimulated a growing body of work. We might suggest consultation of some of the work of Ericksen already cited, James [38-43], Parry [61], and Pitteri [67-71]. More closely connected to this study is the work of Ball and James on fine twinning [6]. Mathematical analysis of the dead loading problem and related issues is due to Fonseca [33] and is briefly noted below. One interesting feature of these ideas is that they may be used to derive the relations of Müller's interaction theory for the ferroelectric transition in Rochelle salt, cf. [47] and [53]. An interesting earlier work about phase transitions is Tisza [77]. The failure of complementing conditions plays a role here. We refer to Simpson and Spector [72] for another view. A summary of the conclusions offered here appears in [46].

First we give a brief description of an elastic crystal and then an example which illustrates some of our concerns. By a (single) crystal we understand a three dimensional lattice given by three independent lattice vectors $\left\{l_{1}, l_{2}, l_{3}\right\}$, written as a matrix $L=\left(l_{1} l_{2} l_{3}\right)$ with columns $\left\{l_{i}\right\}$. Owing to the frame indifference of the Helmholtz free energy $\Phi$ and its invariance under change of lattice basis, cf.[21,23,25,31],

$$
\Phi=\Phi\left(L^{\top} L\right)=\Phi\left(M^{\top} L^{\top} L M\right), \quad M \in G L\left(\mathbb{Z}^{3}\right)
$$

With this fixed reference basis of lattice vectors, the energy density (per unit reference volume) is given by

$$
W(F)=\Phi\left(L^{\top} C L\right), \quad C=F^{\top} F, \quad \operatorname{det} F>0 .
$$

Thus

$$
\begin{gathered}
W(F)=W(Q F H) \quad \text { for } \operatorname{det} F>0, Q^{\top} Q=1, \operatorname{det} Q=1, \\
H \in H,
\end{gathered}
$$

where $H=L G L\left(\mathbb{Z}^{3}\right) L^{-1}$ is a conjugate group of $G L\left(\mathbb{Z}^{3}\right)$. We impose on $W$, or $\Phi$, the conditions

$$
\begin{aligned}
& W(F) \geq 0, \quad W(1)=0, \text { and } \\
& \lim _{\operatorname{detF} \rightarrow 0} W(F)=\infty .
\end{aligned}
$$


that infinite energy be required to compress the body to a point. It is decidedly a hinderance in the analysis and may well be thought of as a constraint. More generally, $\Phi$ may depend as well on temperature, lattice shifts (in the case of more complicated crystals), or polarization (when electromagnetic fields are active.) For the present we do not consider these alternatives.

One question of interest here is to study possible deformations $y(x)$ of a suitable bounded domain $\Omega \subset \mathbb{R}^{n}(n=2$ or 3 ) with the property that

$$
\int_{\Omega} w(\nabla y) d x=\inf \left\{\int_{\Omega} w(\nabla v) d x: v=y_{0} \text { on } a \Omega\right\}
$$

where $y_{0}$ is assigned and the infimum is taken over a suitable class.

Another question concerns possible deformations $y(x)$ realizing extrema of the dead loading problem

$$
\begin{aligned}
& \int_{\Omega} w(\nabla y) d x-\int_{\Omega} f \cdot y d x-\int_{\partial \Omega} g \cdot y d S \\
& =1=\inf \left\{\int_{\Omega} w(\nabla v) d x-\int_{\Omega} f \cdot v d x-\int_{\partial n} g \cdot v d S\right\}
\end{aligned}
$$

This problem has been studied by I. Fonseca [33], who obtained the very interesting result that $l>-\infty$ only if $f=g=0$.

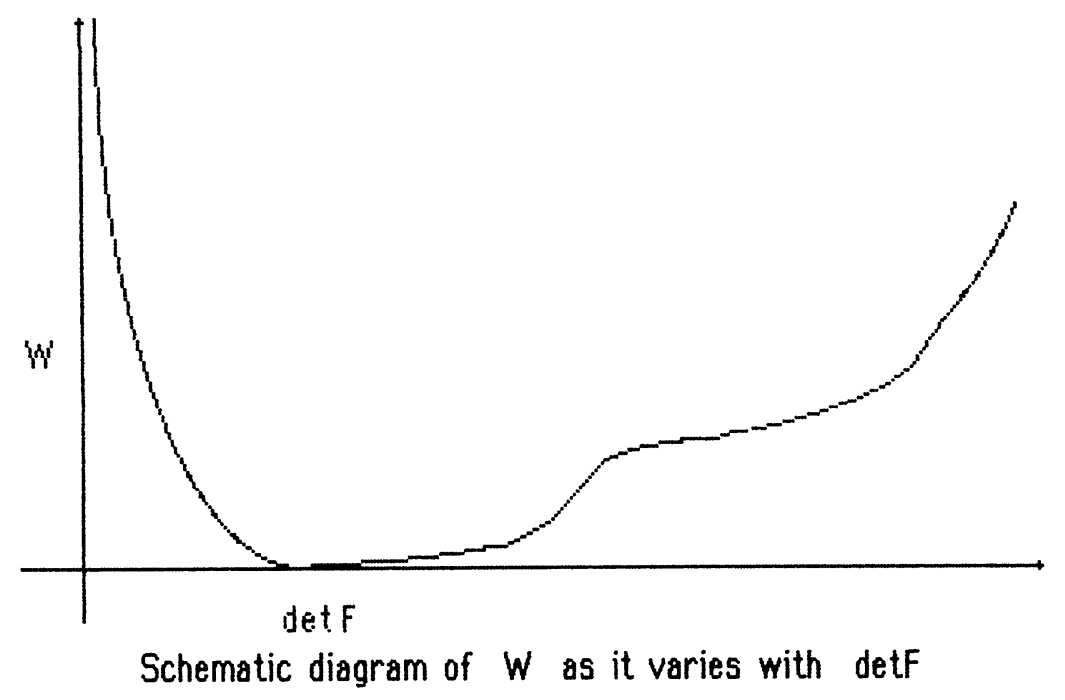




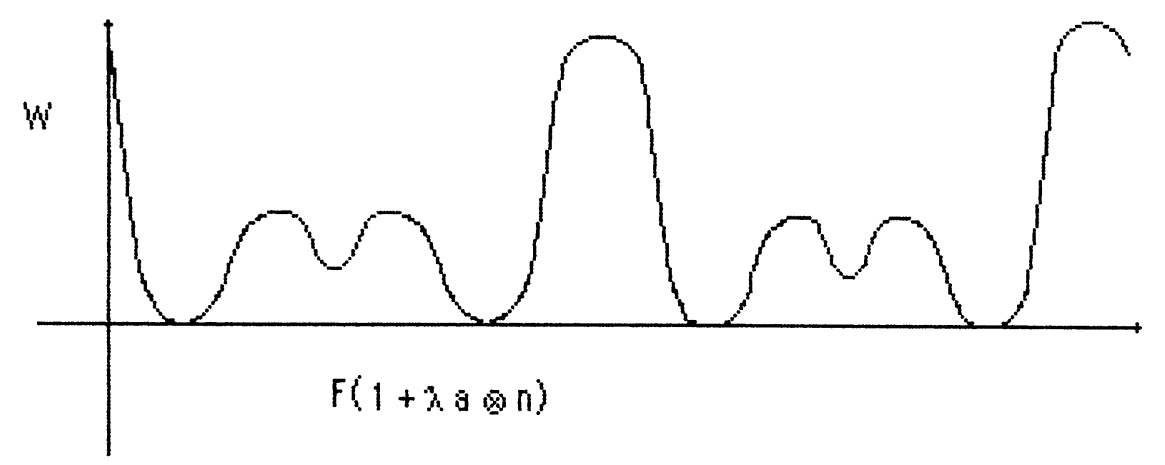

Schematic diagram of the periodic nature of $W$

The analytical difficulties encountered in the study of such $W$ are well publicized, by now, but may withstand a brief review. Note that given $F$, $\operatorname{det} F>0$, and $H=1+a \otimes n \in \mathbb{H}$, a direct calculation shows that the function

$$
W(F(1+\lambda a \otimes n)),-\infty<\lambda<\infty,
$$

is periodic of period one, [33]. In particular this implies that there are points $\lambda$ for which

$$
\begin{aligned}
& c_{i j h k} a^{i} a^{h} n j^{j} n^{k}<0, \\
& c_{i j h k}=\partial^{2} W / \partial F_{i j} \partial F_{h k}(1+\lambda a \oslash n) .
\end{aligned}
$$

Thus $W$ is not rank 1 convex, and in particular, neither quasiconvex nor lower semicontinuous. Indeed, the deformation $1+\lambda a \otimes n$, with $\lambda$ satisfying (1.6), is infinitesimally unstable; whereas, homogeneous deformations are minima of quasiconvex energies by definition. This may also be contrasted with the result of Knops and Stuart [48]. Some recent discussions of the role of quasiconvexity in elasticity have been given by Ball [2-4] and Ball and Murat [7]. The book by Dacarogna [14] provides a treatment of quasiconvexity and lower semicontinuity in general.

A different way to study (1.5) is given by I. Fonseca, as noted earlier. Since the subenergy is a function of $\operatorname{det} \nabla v$ alone, it is useful to consider (1.5) for such $W$. The loadings under which this problem is stable have been considered by I. Fonseca and L. Tartar [36]. Directions in the continuum theory of crystalline solids are discussed in Metastability and incompletely posed problems, I. M. A. Volumes [31]. We refer in 
particular to the articles by Ericksen, James, Kinderlehrer, Pitteri, and Wright. The paper by Ericksen [31] explains the basis for considering stored energy functions exhibiting the invariance we shall discuss.

\section{A useful construction}

To give a brief and simple example, suppose that we are given

$$
\begin{aligned}
& F_{0} \text {, with } \operatorname{det} F_{0}>0, \\
& B=1+a \oplus b \text {, with } \operatorname{det} B=1+a \cdot b>0 \text {, and } \\
& \theta, \quad 0<\theta<1 \text {, }
\end{aligned}
$$

and consider

$$
\begin{aligned}
& y(x)=F x \text { where } \\
& F=F_{0}(1+\theta a \cdot b)=(1-\theta) F_{0}+\theta F_{0} B
\end{aligned}
$$

We assume that $|b|=1$. Let $x(t)$ be the characteristic function of the real interval $(0, \theta)$ in $(0,1)$ and extend it to a 1-periodic function on $\mathbb{R}$. Set

$$
f^{k}(x)=x(k b \cdot x), \quad k=1,2,3, \ldots
$$

Given any bounded domain $\Omega \subset \mathbb{R}^{3}$ it is easy to check that

$$
f^{k} \rightarrow \theta \text { in } L^{\infty}(\Omega) \text { weak *, }
$$

which, we are reminded, means that the averages

$$
|D|^{-1} \int_{D} f^{k} d x \rightarrow \theta \quad \text { for any } D \subset \Omega
$$

or equivalently, that

$$
\int_{\Omega} f^{k} \zeta d x \rightarrow \theta \int_{\Omega} \zeta d x \text { for any } \zeta \in L^{\prime}(\Omega) \text {. }
$$




$$
u(\xi)=F_{0}\left(\xi+\int_{[0, r]} x(t) d t a\right), r=\xi \cdot D
$$

and

$$
u^{k}(x)=k^{-1} u(k x)
$$

it is clear that

$$
u^{k} \rightarrow y \text { in } H^{1, \infty}(\Omega) \text { weak*. }
$$

This means that

$$
\begin{aligned}
& u^{k} \rightarrow y \quad \text { uniformly in } \Omega \text { and } \\
& \begin{aligned}
\nabla u^{k}=F^{k}= & F_{0}\left(1+f^{k} a \otimes b\right)=\left(1-f^{k}\right) F_{0}+f^{k} F_{0} B \\
\rightarrow F & \text { in } L^{\infty}(\Omega) \text { weak*. }
\end{aligned}
\end{aligned}
$$

Now

$$
W\left(F^{k}\right)=\left(1-f^{k}\right) W\left(F_{0}\right)+f^{k} W\left(F_{0} B\right)
$$

whence

$$
W\left(F^{k}\right) \rightarrow(1-\theta) W\left(F_{0}\right)+\theta W\left(F_{0} B\right) \text { in } L^{\infty}(\Omega) \text { weak*. }
$$

Let us analyze this situation in several ways. First choose

$$
F_{0}=1 \text { and } B=H=1+a \otimes n \in \mathbb{H}
$$

so

$$
F^{k}=1+f^{k} a \otimes n
$$

and

$$
W\left(F^{k}\right)=0
$$

However,

$$
W(1+\theta a \oplus n)>0=\lim _{k \rightarrow \infty} W\left(F^{k}\right)
$$

unless by some stroke of luck, $1+\theta a \otimes n \in \mathbb{H}$. 
We have calculated the energy via a minimizing sequence without regard to the numerical value of $W(F)$. We claim, nonetheless, that the successive shearing of a crystal among its natural states should give rise to a distribution of minimum energy, so the final energy density is indeed

$$
W=\lim _{k \rightarrow \infty} W\left(F^{k}\right)=0
$$

In fact, for any continuous function $\psi(F)$,

$$
\bar{\psi}=\lim _{k \rightarrow \infty} \psi\left(F^{k}\right)=(1-\theta) \psi(1)+\theta \psi(1+a \otimes n)
$$

For example, the Piola-Kirchhoff stress

$$
\bar{S}=(1-\theta) S(1)+\theta S(1+a \otimes n)=0,
$$

since $W$ assumes its minimum value at both $F=1$ and $F=H=$ $1+a \otimes n \in \mathbb{H}$. The limit solution may be interpreted in terms of a measure on the space $M$ of matrices with positive determinant given by

$$
v=(1-\theta) \delta_{1}+\theta \delta_{H},
$$

where $\delta_{F}$ denotes the Dirac measure at $F$. So

$$
\begin{aligned}
& \bar{W}=\int_{M} w(A) d v(A)=0, \\
& \bar{S}=\int_{M} S(A) d v(A)=0, \text { and even } \\
& 1+\theta a \otimes n=\int_{M} A d v(A) .
\end{aligned}
$$

As we noted in the introduction, a limit configuration found in this way may be macroscopically homogeneous but infinitely twinned array of states of minimum energy. It would seem that the crystal seeks to return to the lowest energy configuration available to it by undergoing small, kinematically admissible shears.

The evidence of defects like twinning, perhaps not immediately obvious from what has been described so far, is retained by the response of 
the deformed body to small displacements, namely the linearized elasticity tensor, which is given by the formula (2.7) evaluated on the tensor

$$
S^{\prime}(F)=\left(\partial S / \partial F_{1 j}\right)=\left(\partial^{2} W / \partial^{2} F_{1 j}\right)
$$

The form of the tensor $S^{\prime}(F)$ may be available from the symmetry of the particular configuration, $\mathrm{cf}$. [54]. The averaged tensor $\overline{\mathrm{S}}^{\prime}$ may then yield information about the consitution of phases. In a particular application, the homogenized tensor associated to a minimizing sequence may be more appropriate. Some discussion of this is given in [13].

More generally, let $F_{0} \in M$ be arbitrary. The sequence $\left(u^{k}\right)$ of deformations has, as we have said, the property that

$$
u^{k} \rightarrow y \text { in } H^{1, \infty}(\mathbb{Q}) \text { weak *. }
$$

If $\psi(A), A \in \mathbb{M}$, is any continuous function, then

$$
\begin{aligned}
\psi^{k}(x) & =\psi\left(F^{k}\right)=\psi\left(F_{0}\left(1+f^{k} a \oslash n\right)\right) \\
& =\psi\left(F_{0}\left(\left(1-f^{k}\right) 1+f^{k} H\right)\right) \\
& =\left(1-f^{k}\right) \psi\left(F_{0}\right)+f^{k} \psi\left(F_{0} H\right),
\end{aligned}
$$

so for any $\zeta \in L^{\prime}(\mathbb{Q})$,

$$
\int_{Q} \psi\left(F^{k}\right) \zeta d x \rightarrow \int_{Q}\left\{(1-\theta) \psi\left(F_{0}\right)+\theta \psi\left(F_{0} H\right)\right\} \zeta d x
$$

In this way, the sequence $\left(u^{k}\right)$ determines the parametrized measure

$$
v=v_{x}=(1-\theta) \delta_{F_{0}}+\theta \delta_{F_{0} H},
$$

that is,

$$
\int_{\mathbb{Q}} \int_{M} \psi(A) \zeta(x) d v d x=\lim _{k \rightarrow \infty} \int_{\mathbb{Q}} \psi\left(F^{k}\right) \zeta d x, \quad \zeta \in L^{\prime}(\mathbb{Q})
$$


smooth boundary and let us set

$$
\begin{gathered}
E_{\Omega}(\mathrm{F})=\inf _{A(\mathrm{~F})} \int_{\Omega} W(\nabla v) d x, \\
A(\mathrm{~F})=A_{\Omega}(\mathrm{F})=\left\{v \in H^{1, \infty}(\Omega) \text { : } \operatorname{det} \nabla v>0 \text { and } v=y \text { on } \partial \Omega\right\} .
\end{gathered}
$$

We wish to verify that the sequence $\left(u^{k}\right)$ constructed above may be slightly modified so that it lies in $A(\mathrm{~F})$. Let $\Omega^{\prime} \subset \subset \Omega$, that is $\Omega^{\prime}$ is compactly contained in $\Omega$, with $\delta=\operatorname{dist}(\Omega, \Omega)$ and let $\eta \in H^{1, \infty}(\mathbb{Q})$ be a cut off function,

$$
\begin{aligned}
\eta & =1 \text { on } \Omega \backslash \mathbb{Q} \text {, } \\
\eta & =0 \text { in } \Omega \text {, and } \\
|\nabla \eta| & \leq 2 / \delta .
\end{aligned}
$$

Set

$$
v=\eta y+(1-\eta) u^{k} \quad \text { in } \Omega
$$

We calculate that

$$
\begin{aligned}
\nabla v & =\eta F+(1-\eta) F^{k}+\left(y-u^{k}\right) \otimes \nabla \eta \\
& =F_{0}\left(1+\left(f^{k}+\eta\left(\theta-f^{k}\right)\right) a \otimes b\right)+\left(y-u^{k}\right) \otimes \nabla \eta .
\end{aligned}
$$

Note that

$$
\operatorname{det}\left(1+\left(f^{k}+\eta\left(\theta-f^{k}\right)\right) a \otimes b\right)=1+\left(f^{k}+\eta\left(\theta-f^{k}\right)\right) a \cdot b>0 .
$$

For each $m \in \mathbb{N}, m>0$, choose $\delta=1 / m, \Omega^{\prime}=\Omega_{m}$, and $k=k_{m}$ so large that

$$
\begin{aligned}
\operatorname{det} \nabla v & \left.=\operatorname{det} F_{0} \operatorname{det}\left(1+\left(f^{k}+\eta\left(\theta-f^{k}\right)\right) a \oplus b\right)+F_{0}^{-1}\left(y-u^{k}\right) \otimes \nabla \eta\right) \\
& >0
\end{aligned}
$$

and

$$
|\nabla v| \leq 2\left|F_{0}\right||B|
$$

The $v=v^{m}$ have the property 


$$
\begin{aligned}
& v^{m} \in A(F) \text { and } \\
& \left\|v^{m}\right\|_{H^{1, \infty}(\Omega)} \leq \text { Const. }=C
\end{aligned}
$$

Also

$$
\begin{aligned}
\left|\int_{\Omega}\left(\nabla v^{m}-F\right) \zeta d x\right| & \leq\left|\int_{\Omega_{m}}\left(F^{k_{m}}-F\right) \zeta d x\right|+\int_{\Omega-\Omega_{m}}(C+|F|) \zeta d x \\
& \leq\left|\int_{\Omega_{m}}\left(F^{k m}-F\right) \zeta d x\right|+C_{1} \int_{\Omega-\Omega_{m}}|\zeta| d x \\
& \rightarrow 0 \text { as } m \rightarrow \infty .
\end{aligned}
$$

So

$$
v^{m} \rightarrow y \quad \text { in } H^{1, \infty}(\Omega) \text { weak *. }
$$

The same argument applies to the $\psi\left(\nabla v^{m}\right)$ for any continuous $\psi$ on $M$; i.e., we can show that

$$
\psi\left(\nabla v^{m}\right) \rightarrow(1-\theta) \psi\left(F_{0}\right)+\theta \psi\left(F_{0}(1+a \otimes b)\right) \text { in } L^{\infty}(\Omega) \text { weak*. }
$$

Thus $\left(v^{m}\right)$ also determines the parametrized measure $\left(v_{x}\right)$ and, in addition this sequence respects the boundary condition. For example, we may demonstrate this theorem.

Theorem 2.1 Let $\Omega \subset \mathbb{R}^{3}$ have a piecewise smooth boundary and let $\mathrm{F} \epsilon$ M. Then

$$
E_{\Omega}(F) \leq|\Omega| W(F(1-a \otimes n))
$$

whenever $\mathrm{a} \cdot \mathrm{n}=0$.

The property of $\Omega$ necessary to obtain the sequence $\left(\mathrm{v}^{\mathrm{m}}\right)$ is only that $|\partial \Omega|=0$, that is, that the three dimensional measure of $\partial \Omega$ be zero. In this situation $\eta$ may be constructed from the distance function to the complement of $\Omega$ and possible integrals over $\partial \Omega$ vanish. We shall illustrate this more clearly later on in the next section by a different method. 
There is a clear technical advantage to insisting that minimizing sequences satisfy a boundary condition, but there is a disadvantage as well. The original sequence $\left(u^{k}\right)$ may actually correspond to a sequence of minimizers, or equilibrium configurations, which themselves converge to another equilibrium configuration, perhaps providing a clearer idea of the mechanical process under study.

Proof. First assume that $1+a \circledast n=1+\theta \hat{a} \circledast \hat{n}$ where $1+\hat{a} \oplus \hat{n} \in \mathbb{H}$. Then with $\left(v^{m}\right)$ constructed as above,

$$
\begin{aligned}
E_{\Omega}(F) & \leq \lim \int_{\Omega} W\left(\nabla v^{m}\right) d x \\
& =\left\{(1-\theta) W\left(F_{0}\right)+\theta W\left(F_{0}(1+\hat{a} \otimes \hat{n})\right)\right\}|\Omega| \quad(c . f .(2 . \mid 3)) \\
& =\left\{(1-\theta) W\left(F_{0}\right)+\theta W\left(F_{0}\right)\right\}|\Omega| \\
& =W\left(F_{0}\right)|\Omega| \\
& =W(F(1-\theta \hat{a} \otimes \hat{n}))|\Omega| .
\end{aligned}
$$

Finally, to complete the proof, we remove the special hypotheses about the vectors $a$ and $n$. This is trivial. Observe that whenever $p, q$ are orthogonal vectors of integers,

$$
1+L P \otimes L^{-T} Q \in \mathbb{H} \text {. }
$$

Obviously, given $a, n \in \mathbb{R}^{3}, a \cdot n=0$, there is a sequence $p_{k}, q_{k} \in \mathbb{Z}^{3}, p_{k} \cdot q_{k}$ $=0$, and $\theta_{k} \in \mathbb{R}$ such that

$$
a \odot n=\lim _{k \rightarrow \infty} \theta_{k} L p_{k} \odot L^{-T} q_{k}
$$

from which the theorem, that is (2.14), follows by continuity of $W$. QED

\section{Subenergy and homogeneous deformation}

In many situations, the minimum energy of a configuration, $E_{\Omega}(F)$, with, let us say, homogeneous boundary values is its subenergy. The subenergy density of $W(F)$ is given by 


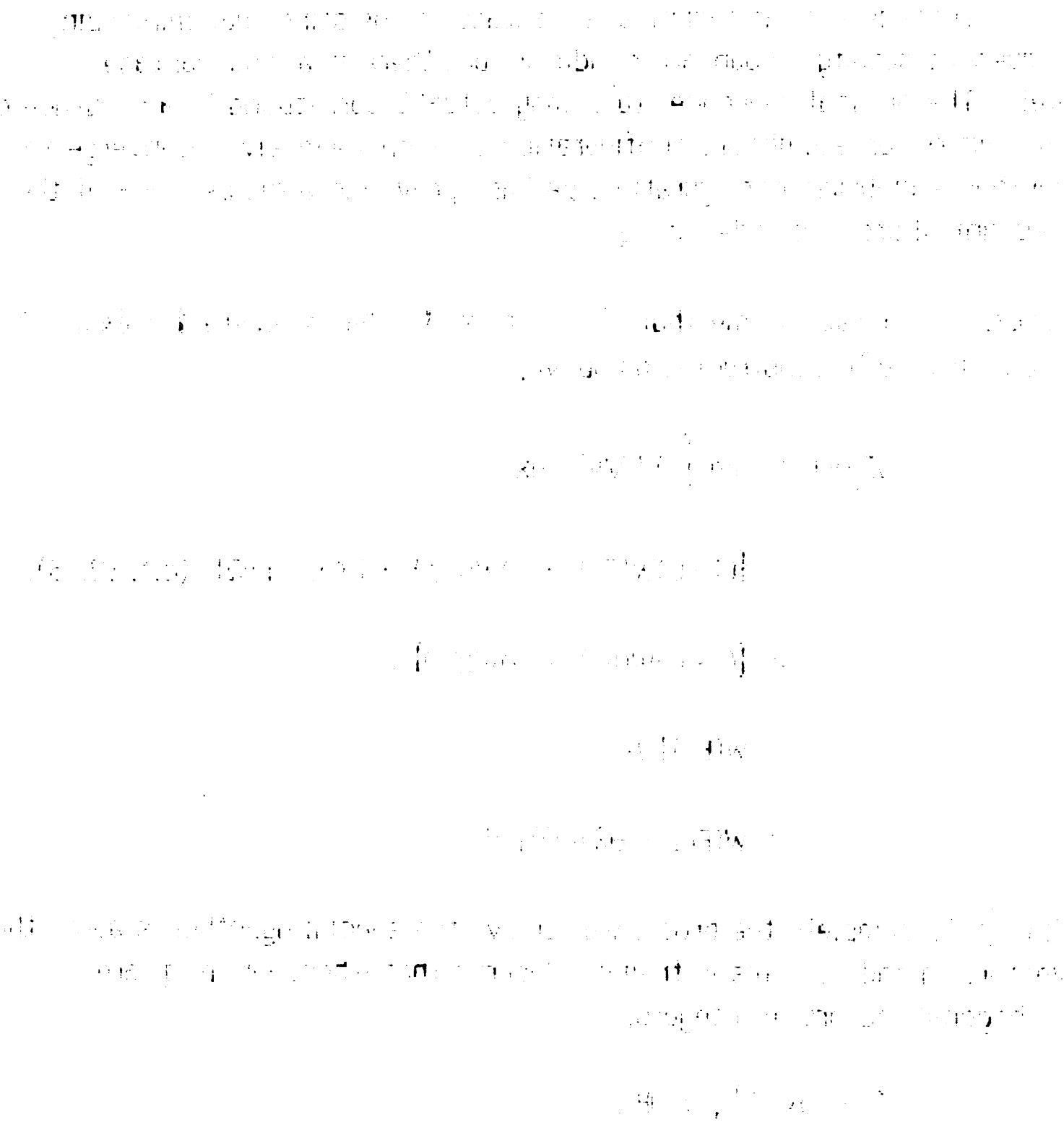

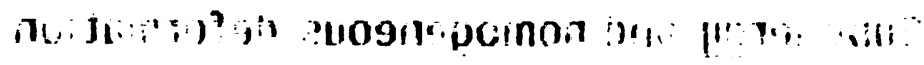

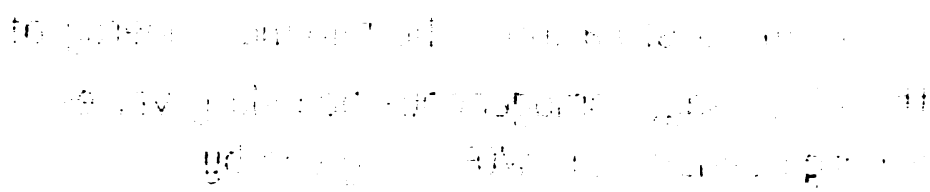


Equilibrium states of crystals

13

$$
\phi(\operatorname{det} F)=\inf _{\operatorname{det} A}=\operatorname{detF} W(A), \quad F \in \mathbb{M} .
$$

We shall also have need of

$$
\phi^{* *}=\text { the convex minorant of } \phi \text {, }
$$

that is, the greatest convex function less than $\phi$. This is shown, roughly speaking, by iterating the argument of the preceding section. The function $\phi$ (jet) may be interpreted as the traditional free energy used by thermodynamicist (cf. Ericksen[24]). We shall establish that it is, in normal regimes, a term used by Ericksen to mean a regime where we may legitamately suppose that $\phi$ is convex, the quasi-convex minorant of $W(F)$. This provides a context for the variational method of seeking minima, which, however, are realized by Young measures rather than classical solutions. More generally, and this may be of interest in the study of thermally driven phase transformations, the minimum energy is achieved by integrating $\phi * *(\operatorname{det} F)$, the convex minorant of $\phi$, over the body.

Let us first note

Proposition 3.1 Let $W$ be given and $\phi$ and $\phi^{* *}$ be defined by (3.1). Then

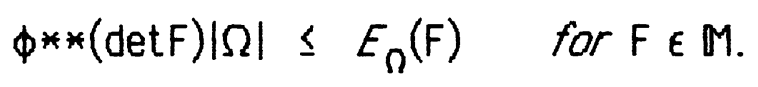

Proof. This follows from Jensen's inequality. First recall that given $\vee \epsilon$ $A(\mathrm{~F})$ [59],

$$
\int_{\Omega} \operatorname{det} \nabla v d x=\int_{\Omega} \operatorname{det} F d x=|\Omega| \operatorname{det} F .
$$

Hence

$$
\begin{aligned}
\int_{\Omega} w(\nabla v) d x & \geq \int_{\Omega} \phi(\operatorname{det} \nabla v) d x \\
& \geq \int_{\Omega} \phi^{* *}(\operatorname{det} \nabla v) d x
\end{aligned}
$$




$$
\begin{aligned}
& \geq|\Omega| \Phi^{* *}\left(|\Omega|^{-1} \int_{\Omega} \operatorname{det} \nabla v d x\right) \\
& \geq|\Omega| \phi^{* *}(\operatorname{det} F) .
\end{aligned}
$$

Our principal result is that equality holds in the conclusion above. We shall proceed in several steps so that we may recover the parametrized measure, at least in some cases, cf. $\$ 4$. The minimum energy admits an elementary scaling property which we wish to elucidate. Given a domain $D$, consider the domain $a+\epsilon D=\left\{x \in \mathbb{R}^{3}: x=a+\epsilon \xi, \xi \in D\right\}$. Then

$$
E_{\mathrm{a}+\epsilon \mathrm{D}}(\mathrm{F})=\epsilon^{3} E_{\mathrm{D}}(\mathrm{F})
$$

This is because a function $\zeta \in A_{a+\epsilon D}(F)$ if and only if

$$
\zeta(x)=\epsilon V\left(\epsilon^{-1}(x-a)\right)+F a \text { for some } V \in A_{D}(F) .
$$

This may be formalized by using an argument of Ball and Murat [7], p. 240. We now prove an intermediate lemma.

Lemma 3.2 There is a function $\tilde{W}(\mathrm{~F})$ such that

$$
E_{0}(F)=|\Omega| \tilde{W}(F)
$$

whenever $\Omega \subset \mathbb{R}^{3}$ is a bounded domain with boundary a $\Omega$ of ( 3 dimensional) measure zero.

Proof. Let $D$ and $\Omega$ be two bounded domains whose boundaries have measure zero. The sets

$$
\{a+\epsilon \bar{D}: a+\epsilon \bar{D} \subset \Omega, a \in \Omega, \epsilon>0\}
$$

form a covering of $\Omega$ to which the Vitali Covering Lemma may be applied. Hence there is a countable or finite subfamily of disjoint subsets

$$
\left\{\bar{D}_{1}=a_{1}+\epsilon_{1} \bar{D}: \bar{D}_{1}=a_{1}+\epsilon_{1} \bar{D} \subset \Omega, a_{1} \in \Omega, \epsilon_{1}>0\right\}
$$

such that 


$$
\Omega=U_{1} \bar{D}_{1} \cup N \quad \text { with }|N|=0 \text {. }
$$

Note that $|\Omega|=\sum \epsilon_{i}^{3}|D|$. Given $\vee \epsilon \quad A_{D}(F)$, define

$$
w(x)=\left\{\begin{array}{cl}
\epsilon_{1} V\left(\epsilon_{i}^{-1}\left(x-a_{1}\right)\right)+F a_{1} & x \in \bar{D}_{1} \\
F x & \text { otherwise }
\end{array}\right.
$$

Then since $\left|\mathrm{\partial D}_{1}\right|=0$,

$$
\begin{aligned}
\int_{\Omega} w(\nabla w) d x & =\Sigma_{1} \int_{D_{1}} w(\nabla w) d x \\
& =\sum_{1} \epsilon_{i}^{3} \int_{D} w(\nabla v) d x \\
& =(|\Omega| /|D|) \int_{D} w(\nabla v) d x .
\end{aligned}
$$

Thus

$$
E_{\Omega}(F) \leq(|\Omega| /|D|) E_{D}(F)
$$

Interchanging the roles of $\Omega$ and $\mathrm{D}$, we obtain that

$$
E_{\Omega}(F) /|\Omega|=E_{D}(F) /|D|
$$

We define

$$
\tilde{W}(F)=E_{D}(F) /|D|
$$

Lemma 3.3 The density $\tilde{W}$ is rank / convex, that is, given

$$
\begin{aligned}
& F, \operatorname{det} F>0, \\
& B=1+a \otimes b, \operatorname{det} B=1+a \cdot b>0 \text {, and } \\
& \theta, \quad 0 \leq \theta \leq 1,
\end{aligned}
$$

then

$$
\tilde{W}((1-\theta) F+\theta F B) \leq(1-\theta) \tilde{W}(F)+\theta \tilde{W}(F B) .
$$

It may be useful to keep in mind that we are actually about to prove that the energy $E_{\Omega}(F)$ is a rank one convex function of $F$. The major 
ingredient of the proof is an energy calculation analogous to the one of the previous section.

Proof. Given $B$, with $|b|=1$, let $D=\mathbb{Q}$ be a unit cube with one face normal to $b$ and determine the sequence $\left(u^{k}\right)$ relative to $F$ as in (2.4). The cube $D$ is then divided into parallel slabs $D_{k, 1}$ of width $1 / k$ and

$$
F^{k}=\nabla u^{k}=F\left(1+f^{k} \mathrm{a}\right) \quad \text { in } D_{k, 1} \text {. }
$$

Each $D_{k, 1}$ may be expressed as $D_{k, 1}=D_{k, i}^{0} \cup D_{k, i}^{1}$ where

$$
f^{k}= \begin{cases}0 & \text { in } D_{k, i}^{0} \\ 1 & \text { in } D_{k, i}^{1}\end{cases}
$$

so

$$
F^{k}=\begin{array}{ll}
F & \text { in } D_{k, i}^{0} \\
& F_{B} \text { in } D_{k, i}^{1} .
\end{array}
$$

Moreover,

$$
\left|\cup D_{k, i}^{0}\right|=(1-\theta)|D|=1-\theta \text { and }\left|\cup D_{k, i}^{1}\right|=\theta
$$

Choose a subsequence of the $\left(u^{k}\right)$, still called $\left(u^{k}\right)$, and a sequence of cut-off functions $\eta^{k}$ such that

$$
v^{k}=\left(1-\eta^{k}\right) y+\eta^{k} u^{k}
$$

have the properties

$$
\begin{aligned}
& v^{k} \in A(F), \operatorname{det} \nabla v^{k}>C>0, \\
& v^{k} \rightarrow y \text { in } H^{1 . \infty}(D) \text { weak*, } \\
& v^{k}=u^{k} \text { in } D^{k},
\end{aligned}
$$

where the $D^{k}$ increase to $D$. There is a sequence $\epsilon_{k} \rightarrow 0$ such that

$$
\int_{\text {supp } \eta^{k}} w\left(\nabla v^{k}\right) d x \leq \epsilon_{k} .
$$

For arbitrary $\zeta \in H_{0}^{1, \infty}\left(D^{k}\right)$, $\operatorname{det}\left(\nabla u^{k}+\nabla \zeta\right)>0$, with 


$$
\zeta=0 \text { on } \partial D_{k, i}^{0} \cup \partial D_{k, i}^{1} \text {, for each } i \text {, }
$$

we have that $\zeta \in H_{0}^{1, \infty}\left(D_{k, i}^{0} \cap D^{k}\right)$ and $\zeta \in H_{0}^{1 . \infty}\left(D_{k, i}^{1} \cap D^{k}\right)$. We may estimate

$$
\begin{aligned}
& E_{D}(F) \leq \int_{D} W\left(\nabla v^{k}+\nabla \zeta\right) d x \\
& \quad \leq \int_{D} W\left(\nabla u^{k}+\nabla \zeta\right) d x+\epsilon_{k} \\
& =\sum_{1} \int_{D_{k, i}^{0}} W(F+\nabla \zeta) d x+\sum_{1} \int_{D_{k, i}^{1}} W(F B+\nabla \zeta) d x+\epsilon_{k} \hat{\alpha}
\end{aligned}
$$

We may take the infimum over $\zeta$, recalling that the infima of the summands are independent of each other. This gives that

$$
E_{D}(F) \leq \Sigma_{1} E_{D_{k, i}^{0} \cap D^{k}}(F)+\Sigma_{i} E_{D_{k, i}^{1} \cap D^{k}}(F B)+\epsilon_{k} .
$$

Applying Lemma 3.2 and noting (3.4),

$$
\begin{aligned}
\tilde{W}(F) & =E_{D}(F) \leq \Sigma_{i}\left|D_{k, i}^{0} \cap D^{k}\right| \tilde{W}(F)+\sum_{i}\left|D_{k, i}^{1} \cap \cap D^{k}\right| \tilde{W}(F B)+\epsilon_{k} \\
& \leq \Sigma_{1}\left|D_{k, i}^{0}\right| \tilde{W}(F)+\Sigma_{1}\left|D_{k, i}^{1}\right| \tilde{W}(F B)+\epsilon_{k} \\
& =(1-\theta) \tilde{W}(F)+\theta \tilde{W}(F B)+\epsilon_{k} .
\end{aligned}
$$

Note that (3.3) may be rewritten in this way. Since for any $F$,

$$
\begin{aligned}
& F=\theta F(1+a \otimes b)+(1-\theta) F(1-(\theta / 1-\theta) a \oplus b), \\
& \tilde{W}(F) \leq \theta \tilde{W}(F(1+a \otimes b))+(1-\theta) \tilde{W}(F(1-(\theta / 1-\theta) a \otimes b)),
\end{aligned}
$$

where $0 \leq \theta \leq 1,1+a \cdot b>0$, and $1-\left({ }_{\theta} / 1-\theta\right) a \cdot b>0$.

Here is a useful fact.

Proposition 3.4 Let $\mathrm{A} \in \mathbb{M}$, i.e., $\mathrm{A}$ is a $3 \times 3$ matrix with positive determinant. Then there is a proper orthogonal $\mathrm{Q}$ and $\mathrm{a}_{1}, \mathrm{n}_{1}, \mathrm{a}_{2}, \mathrm{n}_{2} \in \mathbb{R}^{3}$ with $a_{1} \cdot n_{1}=0, i=1,2$, such that 


$$
A=(\operatorname{det} A)^{1 / 3} Q\left(1+a_{2} \otimes n_{2}\right)\left(1+a_{1} \otimes n_{1}\right) .
$$

If $A$ is a $2 \times 2$ matrix, we may take $a_{2}=n_{2}=0$ and replace the exponent $1 / 3$ by $\frac{1}{2}$.

Many items of this nature may be found in Kelvin and Tait [45], cf. also Love [54], however we did not find this one there.

Proof. Consider the two dimensional case first. Let

$$
E=E_{\delta}=1+\gamma e_{1} \otimes e_{2}, \quad y \in \mathbb{R},
$$

and

$$
\begin{aligned}
U= & E^{\top} E=1+\gamma\left(e_{1} \otimes e_{2}+e_{2} \oplus e_{1}\right)+y^{2} e_{2} \otimes e_{2} \\
= & \left(\begin{array}{cc}
1 & y \\
y & 1+y^{2}
\end{array}\right) .
\end{aligned}
$$

So we may write, by the polar and spectral decompositions,

$$
U=P \Lambda^{2} P^{\top} \text { and } E=R U^{1 / 2}=R P \wedge P^{\top} \text {, }
$$

where $\Lambda$ is diagonal and $R$ and $P$ are proper orthogonal. Thus,

$$
\Lambda=(R P)^{\top} E P=Q(1+a \otimes n)
$$

where $a=y P^{\top} e_{1}$ and $n=P^{\top} e_{2}$.

With $\Lambda=\operatorname{diag}\left(\lambda, \lambda^{-1}\right)$, we determine the relationship between $\lambda$ and $y$. Indeed, since

$$
\begin{aligned}
& \operatorname{det} U=1 \text { and } \operatorname{tr} U=2+y^{2} \\
& y=\lambda-1 / \lambda .
\end{aligned}
$$

Thus given $\lambda>0$, we may find an $E_{\gamma}$ such that (3.7) holds.

Now let $A$ be a $3 \times 3$ matrix with $\operatorname{det} A=1$. Again using the polar and spectral decompositions, 


$$
A=Q S=R P D P^{\top}
$$

where $D=\operatorname{diag}\left(\lambda, \mu,(\lambda \mu)^{-1}\right)$ is diagonal. Thus

$$
\begin{aligned}
D & =\operatorname{diag}\left(\lambda, 1, \lambda^{-1}\right) \operatorname{diag}\left(1, \mu, \mu^{-1}\right) \\
& =R_{1}\left(1+\alpha_{2} \circledast v_{2}\right)\left(1+\alpha_{1} \otimes v_{1}\right),
\end{aligned}
$$

using (3.7) for each of the factors. Substitution of (3.10) into (3.9) and rearranging the rotation gives the result.

QED

More precise information is available using (3.8).

Lemma 3.5 Let $\Omega$ be a bounded domain with $|\partial \Omega|=0$. If $\mathrm{F}$ is $a$ constant matrix with $\operatorname{det} F>0$, then

$$
E_{\Omega}(F) \leq|\Omega| \phi(\operatorname{det} F)
$$

Equality halds in (3.11) if det $\mathrm{F}$ is a point of convexity of $\phi$.

By a point of convexity we intend a point $t$ where $\phi(t)=\phi^{* *}(t)$.

Proof. Given $\epsilon>0$, choose $F_{\epsilon}$, $\operatorname{det} F_{\epsilon}=\operatorname{det} F$, such that

$$
W\left(F_{\epsilon}\right) \leq \phi(\operatorname{det} F)+\frac{1}{2} \epsilon
$$

According to the last proposition we may write

$$
F\left(F_{\epsilon}\right)^{-1}=R\left(1+\alpha_{2} \otimes v_{2}\right)\left(1+\alpha_{1} \otimes v_{1}\right)
$$

which may be rearranged to read

$$
F=R F_{\epsilon}\left(1+a_{2} \circledast n_{2}\right)\left(1+a_{1} \circledast n_{1}\right)
$$

Now we may find $\hat{a}_{1}, \hat{n}_{1}$, and $\theta_{1}, i=1,2$, with $H_{1}=1+\hat{a}_{1} \otimes \hat{n}_{1} \in \mathbb{H}$, such that

$$
\left(1+a_{2} \odot n_{2}\right)\left(1+a_{1} \odot n_{1}\right)=(1+\Delta)\left(1+\theta_{2} \hat{a}_{2} \odot \hat{n}_{2}\right)\left(1+\theta_{1} \hat{a}_{1} \odot \hat{n}_{1}\right)
$$

or 


$$
F=Q F_{\epsilon}(1+\Delta)\left(1+\theta_{2} \hat{a}_{2} \otimes \hat{n}_{2}\right)\left(1+\theta_{1} \hat{a}_{1} \oplus \hat{n}_{1}\right)
$$

with $|\Delta|$ so small that

$$
\left|W\left(F_{\epsilon}(1+\Delta)\right)-W\left(F_{\epsilon}\right)\right|<\frac{1}{2} \epsilon .
$$

For convenience, let us set $H_{1}=1+\hat{a}_{1} \otimes \hat{n}_{1}, \quad i=1,2$. By Lemma 3.3,

$$
\begin{aligned}
E_{\Omega}(F)= & E_{\Omega}\left(Q F_{\epsilon}(1+\Delta)\left(1+\theta_{2} \hat{a}_{2} \otimes \hat{n}_{2}\right)\left(1+\theta_{1} \hat{a}_{1} \otimes \hat{n}_{1}\right)\right) \\
\leq & \left(1-\theta_{1}\right)\left(1-\theta_{2}\right) E_{\Omega}\left(Q F_{\epsilon}(1+\Delta)\right)+ \\
& \left(1-\theta_{1}\right) \theta_{2} E_{\Omega}\left(Q F_{\epsilon}(1+\Delta) H_{2}\right)+\theta_{1}\left(1-\theta_{2}\right) E_{\Omega}\left(Q F_{\epsilon}(1+\Delta) H_{1}\right)+ \\
& \theta_{1} \theta_{2} E_{\Omega}\left(Q F_{\epsilon}(1+\Delta) H_{2} H_{1}\right) \\
\leq & W\left(F_{\epsilon}(1+\Delta)\right)|\Omega| \\
\leq & W\left(F_{\epsilon}\right)|\Omega|+\frac{1}{2} \epsilon|\Omega| \\
\leq & (\phi(\operatorname{det} F)+\epsilon)|\Omega| . \quad \text { QED }
\end{aligned}
$$

We may now make a general statement about homogeneous deformations.

Theorem 3.6 Let $\Omega$ be a domain with boundary a $\Omega$ of (three dimensional) measure zero. Then

$$
\tilde{W}(F)=|\Omega|^{-1} E_{\Omega}(F)=\phi^{* *}(\operatorname{det} F), \text { for } F \in M \text {. }
$$

Proof. In view of Lemma 3.5, note (3.11), we must check (3.12) at points where $\phi^{* *}(\operatorname{det} F)<\phi(\operatorname{det} F)$. Suppose first that for such $F$ there are numbers $v_{1}$ and $v_{2}$ and $\theta \in(0,1)$ such that

$$
\begin{aligned}
& \operatorname{det} F=\theta v_{1}+(1-\theta) v_{2} \\
& \phi\left(v_{i}\right)=\phi^{* *}\left(v_{i}\right), i=1,2 .
\end{aligned}
$$

Choose $a, b \in \mathbb{R}^{3}$ such that

which implies that

$$
v_{1}=\operatorname{det} F(1+a \cdot b)
$$




$$
v_{2}=\operatorname{detF}(1-(\theta / 1-\theta) a \cdot b) .
$$

By Lemma 3.2, Lemma 3.5, and (3.5),

$$
\begin{aligned}
& \phi * *(\operatorname{det} F) \leq \tilde{W}(F) \\
& \leq \theta \tilde{W}(F(1+a \oplus b))+(1-\theta) \tilde{W}\left(F\left(1-\left(\theta / /_{-\theta}\right) a \oplus b\right)\right) \\
& \leq \theta \phi(\operatorname{det} F(1+a \cdot b))+(1-\theta) \phi(\operatorname{det} F(1-(\theta / 1-\theta) a \cdot b)) \\
& =\phi^{* *}(\operatorname{det} F) \text {. }
\end{aligned}
$$

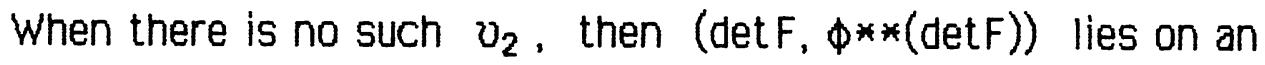
asymptotic direction of the graph of $\phi^{* *}$. In this case there is a $v_{1}$, $0<v_{1}<\operatorname{det} F$, such that $\phi\left(v_{1}\right)=\phi * *\left(v_{1}\right)$,

$$
\phi * *(v)=\phi\left(v_{1}\right)+p\left(v-v_{1}\right), \text { for } v>v_{0} \text {, }
$$

with

$$
p=\lim _{v \rightarrow \infty}\left(\phi(v)-\phi\left(v_{1}\right)\right) /\left(v-v_{1}\right)
$$

Moreover,

$$
\phi * *(v) \leq \phi(v)
$$

As before, choose $a, b \in \mathbb{R}^{3}$ such that $v_{1}=\operatorname{det} F(1+a \cdot b)$. Set

$$
F_{k}=F(1-(k-1) a \otimes b), k \geq 0
$$

Note that $\operatorname{det} F_{0}=v_{1}$. Consequently we may write

$$
F=\theta F_{0}+(1-\theta) F_{k} \text { for } \theta=\theta_{k}=(k-1) / k, k \geq 1,
$$

so that

$$
\begin{aligned}
\phi * *(\operatorname{det} F) & \leq \theta \tilde{W}\left(F_{0}\right)+(1-\theta) \tilde{W}\left(F_{k}\right) \\
\leq & \theta \phi\left(\operatorname{det} F_{0}\right)+(1-\theta) \phi\left(\operatorname{det} F_{k}\right) \\
& =\phi\left(\operatorname{det} F_{0}\right)+ \\
& \quad(1-\theta)\left\{\phi\left(\operatorname{det} F_{0}-k \operatorname{det} F a \cdot b\right)-\phi\left(\operatorname{det} F_{0}\right)\right\}
\end{aligned}
$$




$$
\begin{aligned}
& =\phi\left(v_{1}\right)+\left\{\phi\left(\operatorname{det} F_{0}-k \operatorname{det} F a \cdot b\right)-\phi\left(\operatorname{det} F_{0}\right) / k\right\} \\
& \rightarrow \phi\left(v_{1}\right)-p \operatorname{det} F a \cdot b=\phi^{* *}(\operatorname{det} F)
\end{aligned}
$$

\section{General boundary conditions and existence of parametrized measures}

In the last section we have seen how an elementary variational argument using little more than the definition of weak* convergence allowed us to find minimum values of energy associated to homogeneous boundary conditions. Here we extend those results in two directions by exploiting very much the same methods. First we shall discuss more general boundary conditions and then we shall consider also some special homogeneous configurations admitting parametrized measure minima.

Given a domain $\Omega \subset \mathbb{R}^{3}$ with Lipschitz boundary $\partial \Omega$ and $y_{0}: \Omega \rightarrow$ $\mathbb{R}^{3}$ a Lipschitz deformation, we set

$$
A_{\Omega}\left(y_{0}\right)=\left\{v \in H^{1.00}(\Omega) ; \operatorname{det} \nabla v>0 \text { and } v=y_{0} \text { on } \partial \Omega\right\}
$$

and

$$
E_{n}\left(y_{0}\right)=\inf _{A\left(y_{0}\right)} \int_{0} w(\nabla v) d x .
$$

Recall that if $y_{0}(x)=y(x)=F x+a$ is an affine transformation, then by Theorem 3.6,

$$
E_{\Omega}\left(y_{0}\right)=\phi^{* *}(\operatorname{det} F)|\Omega|
$$

where $\phi^{* *}$ is the convexification of the subenergy of $W, c f .(3.1)$. For mappings of this form we retain our old notations when convenient, that is

$$
A_{\Omega}(\mathrm{F} x)=A_{\Omega}(\mathrm{F}) \text { and } E_{\Omega}(\mathrm{F} x)=E_{\Omega}(\mathrm{F})
$$

Our results may be extended to more general boundary conditions. We give an example of this.

Theorem 4.1 Let $\Omega \subset \mathbb{R}^{3}$ be a bounded domain with Lipschitz boundary and $y_{0} \in C^{1}(\bar{\Omega}), \operatorname{det} \nabla y_{0} \geq c>0$. Suppose that 


$$
\inf _{A\left(y_{0}\right)} \int_{\Omega} \phi^{* *}(\operatorname{det} \nabla v) d x=\inf _{A\left(y_{0}\right) n c^{\prime}(\overline{)})} \int_{\Omega} \phi^{* *(\operatorname{det} \nabla v) d x}
$$

Then

$$
E_{\Omega}\left(y_{0}\right)=\inf _{A\left(y_{0}\right)} \int_{\Omega} \phi^{* *}(\operatorname{det} \nabla v) d x
$$

Proof. Since $\phi^{* *}(\operatorname{det} A) \leq W(A)$ for $A \in \mathbb{M}$, it is obvious that

$$
E_{\Omega}\left(y_{0}\right) \geq \inf _{A\left(y_{0}\right)} \int_{\Omega} \phi * *(\operatorname{det} \nabla v) d x .
$$

We need to prove only that

$$
E_{\Omega}\left(y_{0}\right) \leq \inf _{A\left(y_{0}\right)} \int_{\Omega} \phi * *(\operatorname{det} \nabla v) d x .
$$

Let $y \in A\left(y_{0}\right) \cap C^{\prime}(\bar{\Omega})$. It is elementary to check that we may find a sequence $\left(w^{k}\right) \subset H^{1, w}(\Omega)$ such that each $w^{k}$ is piecewise affine and

$$
\begin{aligned}
& w^{k} \rightarrow y \quad \text { in } H^{1, \infty}(\Omega) \text { weak* and } \\
& \nabla w^{k} \rightarrow \nabla y \quad \text { uniformly in } \bar{\Omega} \text {, }
\end{aligned}
$$

cf. Ekeland and Temam [19], Proposition 2.1, p.309, for example. In particular,

$$
\operatorname{det} \nabla w^{k} \rightarrow \operatorname{det} \nabla y \quad \text { uniformly in } \bar{\Omega}
$$

Indeed, a neighborhood of $\bar{\Omega}$ may be written as the union of simplices $D_{k, j}$ of diameter $\leq 1 / k$ and for each $k$ we may express

$$
F^{k}=\nabla w^{k}=\Sigma_{j} F^{k, j} \chi_{k, j}
$$

where $x_{k, j}$ is the characteristic function of the simplex $D_{k, j}$ and the $F^{k . j}$ are constant matrices with positive determinant for $k$ sufficiently large.

According to Theorem 3.6, given $\epsilon>0$, for each $k$ we may find a $u^{k . j}$ with

$$
u^{k, J}=w^{k} \text { on } \partial D_{k, j} \quad \text { and }
$$




$$
\int_{D_{k, j}} W\left(\nabla u^{k, j}\right) d x \leq \phi^{* *}\left(\operatorname{det} F^{k, j}\right)\left|D_{k, j}\right|+\epsilon Z^{-j}
$$

Moreover, for given $\epsilon>0$, we may choose $k$ so large that

$$
\left|\phi^{* *}\left(\operatorname{det} F\left(x_{k, j}\right)\right)-\phi^{* *}\left(\operatorname{det} F^{k, J}\right)\right|<\epsilon .
$$

where $x_{k, j}$ is the barycenter of $D_{k, j}$ and $F=\nabla y$. Thus

$$
\int_{\Omega} W\left(\nabla u^{k}\right) d x \leq \sum_{j} \phi^{* *}\left(\operatorname{det} F\left(x_{k, j}\right)\right)\left|D_{k, j}\right|+\epsilon|\Omega|+\epsilon .
$$

Since the first term on the right hand side is a Riemann sum for

$$
\int_{\Omega} \phi^{* *(\operatorname{det} F)) d x}
$$

we conclude that

$$
\left.\lim _{\mathrm{inf}} \int_{\Omega} w\left(\nabla u^{k}\right) d x \leq \int_{\Omega} \phi^{* *}(\operatorname{det} F)\right) d x
$$

To complete the argument we must show that the boundary conditions on the admissible functions are not too restrictive. Given $\delta>0$, let $\Omega_{\delta}$ denote the union of the simplices $D_{k_{1} j}$ of distance to $\partial \Omega$ greater than $\delta$. It is elementary to check that

$$
\lim _{\delta \rightarrow 0}\left|\Omega-\Omega_{\delta}\right|=0
$$

Let $\eta_{\delta}$ be a Lipschitz function with $\eta_{\delta}=1$ on $\partial \Omega, \eta_{\delta}=0$ on $\Omega_{\delta}$, and $0 \leq \eta_{\delta} \leq 1,\left|\nabla \eta_{\delta}\right| \leq$ const. $/ \delta$, where the constant may be taken independent of $k$. Let $u^{k}$ denote the function equal to $u^{k, J}$ in $D_{k_{1} J}$ determined above, and define $v^{k} \in A\left(y_{0}\right)$ by

$$
v^{k}= \begin{cases}\left(1-\eta_{\delta}\right) y+\eta_{\delta} w^{k} & \text { in } \Omega-\Omega_{\delta} \\ u^{k} & \text { in } \Omega_{\delta}\end{cases}
$$

with $k$ chosen so large that

$$
\left|\nabla v^{k}\right| \leq \text { Const. and in } \Omega-\Omega_{\delta}
$$




$$
\text { det } \nabla v^{k} \geq \frac{1}{2} c>0
$$

which is possible by (4.6) and (4.7). This implies that

$$
\int_{\Omega-\Omega_{\delta}} W\left(\nabla v^{k}\right) d x \leq C\left|\Omega-\Omega_{\delta}\right| \text { for a constant } C>0 \text {. }
$$

We may now apply (4.9), with fixed $\delta$, whence

$$
\begin{aligned}
& E_{\Omega}\left(y_{0}\right) \leq \liminf _{k \rightarrow \infty} \int_{\Omega} w\left(\nabla v^{k}\right) d x \\
& \leq \liminf _{k \rightarrow \infty} \int_{\Omega} w\left(\nabla u^{k}\right) d x+c\left|\Omega-\Omega_{\delta}\right| \\
& \left.\leq \int_{\Omega} \phi^{* *}(\operatorname{det} F)\right) d x+C\left|\Omega-\Omega_{\delta}\right| .
\end{aligned}
$$

The result follows from (4.10).

The condition (4.4) may be regarded as a technical hypothesis. We do not know at this writing a way to approximate arbitrary admissible Lipschitz deformations by piecewise affine ones. On the other hand, there are many circumstances where (4.4) may be verified. For example, if the right hand side of (4.4) is zero. More generally, we note

Theorem 4.2 Let $\Omega$ be a bounded Lipschitz domain and let $y_{0} \in C^{\prime}(\bar{\Omega})$ satisfy det $\nabla y_{0}=v$, a constant. Then

$$
E_{\Omega}\left(y_{0}\right)=\phi^{* *}\left(\operatorname{det} \nabla y_{0}\right)|\Omega| .
$$

Proof. The proof is another application of Jensen's inequality almost precisely as in Proposition 3.1 noting that in this case (4.4) is satisfied since

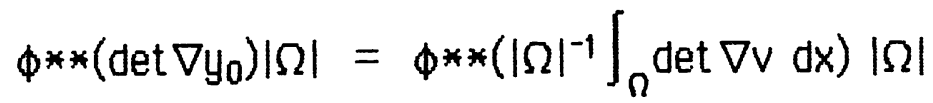

$$
\begin{aligned}
& \leq \inf _{A\left(y_{0}\right)} \int_{\Omega} \phi^{* *}(\operatorname{det} \nabla v) d x \\
& \leq \inf _{A\left(y_{0}\right) n c^{\prime}(n)} \int_{\Omega} \phi * *(\operatorname{det} \nabla v) d x
\end{aligned}
$$




$$
\leq \phi * *\left(\operatorname{det} \nabla y_{0}\right)|\Omega| .
$$

QED

Indeed, we observe that there are many such mappings. For example, as Tartar (cf. [36]) has pointed out, in two dimensions the mapping of the unit disc given in polar coordinates by

$$
r=\rho, \quad \Theta=\theta+\zeta(p)
$$

where $\zeta$ is any smooth function with support in $(0,1)$, has constant determinant one. Another way to approach this question is through a result of Mascolo and Schianchi [56], using a theorem of Moser's [60].

There are additional situations where (4.4) may be verified. For example, let $\Omega$ be the square

and

$$
\Omega=\left\{x_{i}\left|x_{1}\right|<1 \text { and } \lambda-1<x_{2}<\lambda\right\}, 0<\lambda<1 \text {, }
$$

$$
\begin{aligned}
& y_{0}(x)=F x \quad \text { with } \\
& F=\left\{\begin{array}{ll}
1+b \otimes \mathrm{e}_{2} & x_{2}>0 \\
1 & x_{2}<0
\end{array},\right.
\end{aligned}
$$

where $b \cdot e_{2} \neq 0$. We may construct a sequence $v^{k} \in A(y)$ such that

$$
\begin{array}{ll}
0<\operatorname{det} \nabla v^{k}<\text { const. } & \text { and } \\
\operatorname{det} \nabla v^{k} \rightarrow 1+\lambda b \cdot e_{2} & \text { a.e. in } \Omega .
\end{array}
$$

Thus

$$
\lim _{k \rightarrow \infty} \int_{\Omega} \phi^{* *}\left(\operatorname{det} \nabla v^{k}\right) d x=\phi * *\left(1+\lambda b \cdot e_{2}\right) .
$$

Keeping in mind that

$$
\begin{aligned}
& \inf _{A\left(y_{0}\right)} \int_{\Omega} \phi^{* *}\left(\operatorname{det} \nabla v^{k}\right) d x \geq \phi^{* *}\left(\int_{\Omega} \operatorname{det} F d x\right) \\
& =\phi^{* *\left(1+\lambda b \cdot e_{2}\right),}
\end{aligned}
$$


deformations agrees with the infimum over Lipschitz ones.

Some additional discussion of related issues may be found in Dacorogna and Fusco [15] and Marcellini [55].

When $F$ is related by symmetry to the minimizer of the subenergy, cf. (4.16) below, we may use the method of $\$ 2$ to construct a parametrized measure, or Young measure, minimum. Let us explain this before passing to more general considerations. Restricting our attention to homogeneous deformations, we introduce a few conventions. Let us say that $\left(v_{x}\right)_{x \in \Omega}$ is a parametrized measure in $\Omega$ satisfying the boundary condition $y=F x$ provided there is a sequence $\left(v^{k}\right) \subset H^{1, \infty}(\Omega)$ such that

$$
\begin{array}{ll}
v^{k} \in A_{\Omega}(y) & \\
\operatorname{det} \nabla v^{k}>0 & \text { in } \Omega \\
v^{k} \rightarrow z & \text { in } H^{1, \infty}(\Omega) \text { weak* }
\end{array}
$$

where

and

$$
z=y \quad \text { on } \partial \Omega \text {, }
$$

$$
\int_{\Omega} \int_{M} \psi(x, A) d v_{x} d x=\lim _{k \rightarrow \infty} \int_{\Omega} \psi\left(x, \nabla v^{k}\right) d x \text { for } \psi \in C(\Omega \times M) .
$$

A parametrized measure $\left(v_{x}\right)_{x \in \Omega}$ satisfying the boundary condition $y=F x$ is a minimum in $\Omega$ if

$$
E_{\Omega}(F)=\int_{\Omega} \int_{M} W(A) d v_{x} d x
$$

Theorem 4.3 Let $\Omega$ be open and bounded with $|a \Omega|=0$ and suppose that $\operatorname{det} \mathrm{F}$ is a point of convexity of $\phi$. Assume that $\mathrm{F} \in \mathbb{M}$ admits the representation

$$
\begin{aligned}
& F=F_{0}\left(1+\theta_{2} \hat{a}_{2} \oplus \hat{n}_{2}\right)\left(1+\theta_{1} \hat{a}_{1} \oplus \hat{n}_{1}\right) \text {, where } \\
& H_{1}=1+\hat{a}_{1} \otimes \hat{n}_{1} \in \mathbb{H}, \quad 0 \leq \theta_{1} \leq 1, \quad i=1,2,
\end{aligned}
$$

where $W\left(F_{0}\right)=\phi\left(\operatorname{det} F_{0}\right)$. Then 


$$
v=v_{x}=\left(1-\theta_{1}\right)\left(1-\theta_{2}\right) \delta_{F_{0}}+\theta_{1}\left(1-\theta_{2}\right) \delta_{F_{0} H_{1}}+\left(1-\theta_{1}\right) \theta_{2} \delta_{F_{0} H_{2}}+\theta_{1} \theta_{2} \delta_{F_{0} H_{2} H_{1}}
$$

is a parametrized measure minimum in $\Omega$.

Note that (4.14) has the special property that

$$
F=\int_{0} A d v
$$

so that $z=y$ in (4.13).

Proof: We consider first the case that $\Omega=\mathbb{Q}$, a cube, since any bounded domain may be enclosed in some such $\mathbb{Q}$ and delay discussion of the boundary condition on $\partial Q$. Choose $\mathbb{Q}$ with faces parallel to $\hat{a}_{1}$ and $\hat{n}_{1}$. Let $F_{1}=F_{0}\left(1+\theta_{2} \hat{a}_{2} \otimes \hat{n}_{2}\right)$ and determine the sequence $\left(u^{k}\right)$ relative to $F_{1}, c f$. (2.4). For each $k$ the cube $Q$ is divided into slabs $D_{1}=D_{k, 1}$ of width $1 / k$ and

$$
\nabla u^{k}=F^{k}=F_{1}\left(1+f^{k} \hat{a}_{1} \otimes \hat{n}_{1}\right) \text { in } D_{1} \text {. }
$$

As in the proof of Lemma 3.3, each $D_{1}=D_{k, i}^{0} \cup D_{k, i}^{1}$, where

$$
f^{k}= \begin{cases}0 & \text { in } D_{k, i}^{0} \\ 1 & \text { in } D_{k, i}^{1}\end{cases}
$$

so

$$
F^{k}= \begin{cases}F_{1} & \text { in } D_{k, i}^{0} \\ F_{1} H_{1} & \text { in } D_{k, i}^{1} .\end{cases}
$$

In particular, $u^{k}$ is affine on each of $D_{k, i}^{0}$ and $D_{k, i}^{1}$. Also,

$$
\left|D_{k, i}^{0}\right|=\left(1-\theta_{1}\right)\left|D_{1}\right| \text { and }\left|D_{k, i}^{1}\right|=\theta_{1}\left|D_{1}\right| \text {. }
$$

For fixed $k, i$ consider the sequence

$$
w^{j, 0} \in H^{1, \infty}\left(D_{k, i}^{0}\right), \quad j=1,2,3, \ldots
$$

constructed in (2.12) satisfying 
Equilibrium states of crystals

29

$$
\begin{aligned}
& w^{1.0}=u^{k} \text { on } 2 D_{k, i}^{0} \\
& w^{1.0} \rightarrow u^{k} \text { in } H^{1, \infty}\left(D_{k, i}^{0}\right) \text { weak* } \\
& \left|\nabla w^{1.0}\right| \leq C \text { and } \operatorname{det} \nabla w^{1.0} \geq c>0 .
\end{aligned}
$$

The sequence $\left(w^{\mathrm{j} .0}\right)$ determines the parametrized measure

$$
v^{0}=\left(1-\theta_{2}\right) \delta_{F_{0}}+\theta_{2} \delta_{F_{0} H_{2}} \text { in } D_{k, i}^{0} \text {. }
$$

Analogously, consider the sequence

$$
w^{j .1} \in H^{1, \infty}\left(D_{k, i}^{1}\right), j=1,2,3, \ldots
$$

constructed in (2.12) satisfying

$$
\begin{aligned}
& w^{j \cdot 1}=u^{k} \text { on } \partial D_{k, i}^{1} \\
& w^{j \cdot 1} \rightarrow u^{k} \text { in } H^{1, \infty}\left(D_{k, i}^{1}\right) \text { weak* } \\
& \left|\nabla w^{j .1}\right| \leq C \text { and } \operatorname{det} \nabla w^{j .1} \geq c>0 .
\end{aligned}
$$

The sequence $\left(w^{\text {d.1 }}\right)$ determines the parametrized measure

$$
v^{\prime}=\left(1-\theta_{2}\right) \delta_{\mathrm{F}_{0} \mathrm{H}_{1}}+\theta_{2} \delta_{\mathrm{F}_{0} \mathrm{NH}_{2}} \text { in } \mathrm{D}_{0, \mathrm{i}}^{1} \text {. }
$$

where $H_{1} N=H_{2} H_{1}$, ie., $N=H_{1}^{-1} H_{2} H_{1} \in \mathbb{H}$. Thus

$$
v^{1}=\left(1-\theta_{2}\right) \delta_{\mathrm{F}_{0} \mathrm{H}_{1}}+\theta_{2} \delta_{\mathrm{F}_{0} \mathrm{H}_{2} \mathrm{H}_{1}} \text { in } \mathrm{D}_{0, i}^{1} \text {. }
$$

Now let $\psi \in C(M)$ and $\epsilon>0$. Let $N=N(K)$ denote the number of slabs and $w^{k, j}$ the function which is $w^{j, 0}$ in $D_{k, i}^{0}$ and $w^{j .1}$ in $D_{0, i}^{1}$ for each $i$. Then for $j$ sufficiently large,

$$
\begin{gathered}
\left|\int_{D_{k, i}^{0} \psi} \psi\left(\nabla w^{k, j}\right) d x-\int_{D_{k, i}^{0} j} \int_{M} \psi(A) d v^{0} d x\right|= \\
\left|\int_{D_{k, i}^{0} \psi} \psi\left(\nabla w^{k, j}\right) d x-\left(\left(1-\theta_{2}\right) \psi\left(F_{0}\right)+\theta_{2} \psi\left(F_{0} H_{2}\right)\right)\right| D_{k, i}^{0} \| \leq \epsilon / 4 N
\end{gathered}
$$

and

$$
\left|\int_{D_{k, i}^{1},} \psi\left(\nabla w^{k, j}\right) d x-\int_{D_{k, i}^{1}} \int_{M} \psi(A) d v^{\prime} d x\right|=
$$




$$
\left|\int_{D_{k, i}^{1},} \psi\left(\nabla w^{k, J}\right) d x-\left(\left(1-\theta_{2}\right) \psi\left(F_{0} H_{1}\right)+\theta_{2} \psi\left(F_{0} H_{2} H_{1}\right)\right)\right| D_{k, i}^{1}|| \leq \epsilon / 4 N .
$$

Summing these two pieces, we obtain that

$$
\left|\int_{D_{k, j}} \psi\left(\nabla w^{k, j}\right) d x-\int_{D_{k, j}} \int_{M} \psi(A)\left(x_{D_{k, j}^{0}} d v^{0}+x_{D_{k, j}^{1},} d v^{1}\right) d x\right| \leq \epsilon / 2 N .
$$

We sum this over i, whence

$$
\left|\int_{Q} \psi\left(\nabla w^{k_{j} j}\right) d x-\int_{0} \int_{M} \psi(A) \sum_{i}\left(x_{D_{k, i}^{0}} d v^{0}+x_{D_{k, i}^{1}} d v^{1}\right) d x\right| \leq \epsilon / 2 .
$$

Now

$$
\begin{aligned}
& \Sigma_{1} x_{D_{k, i}^{0}}=1-f^{k} \rightarrow 1-\theta_{1} \text { and } \\
& \sum_{1} x_{D_{k, i}^{1}}=f^{k} \rightarrow \theta_{1}
\end{aligned}
$$

so for $k$ and $j$ sufficiently large,

$$
\left|\int_{Q} \psi\left(\nabla w^{k, j}\right) d x-\iint_{Q} \psi(A) d v d x\right|<\epsilon .
$$

Since $C(M)$ is separable, we may choose a subsequence of the $w^{k . j}$, which we refer to as $\left(v^{k}\right)$, with the property that

$$
\int_{M} \psi(A) d v|\mathbb{Q}|=\lim _{k \rightarrow \infty} \int_{\Omega} \psi\left(\nabla v^{k}\right) d x \text { for all } \psi \in C(M)
$$

To show that (4.23) implies (4.14) is a routine affair. By scaling and translating $\left(v^{k}\right),(4.23)$ is seen to hold for a cube of any dimensions and center. It follows that (4.14) holds for functions $\psi(x, A)$ of the form

$$
\psi(x, A)=\sum_{1 \leq i \leq N} C_{1} x_{Q_{1}}(x) \psi_{1}(A)
$$

where the $\mathbb{Q}_{i}$ are disjoint cubes and the $\psi_{1} \in C(\mathbb{M})$. From this and the separability of $C(\Omega \times M)$, one infers the existence of a sequence $\left(v^{k}\right)$ for 
which (4.14) holds.

Finally, suppose that $\Omega \subset \mathbb{Q}$ is an open set with $|\partial \Omega|=0$. We may adjust the boundary behavior of our approximating sequence by asking that $w^{k . J}=u^{k}$ in a small neighborhood of $\partial D_{k, 1} \cap \partial \Omega$ and then interpolate between $u^{k}$ and Fx. This may be achieved because $\nabla\left(w^{k, j}-u^{k}\right)$ and $F-$ $\nabla u^{k}$ are rank one matrices. The argument is identical to $\$ 2$ or Lemma 3.3 .

QED

The measure we have found is not unique, which will be obvious from the next section. In a subsequent paper we shall show how our methods may be used to determine parametrized measures for any homogeneous configuration. The matrices satisfying (4.16) are dense in all matrices, and this is sufficient to determine energy, as we have seen, and stress also, but it is not satisfactory to determine, for example, linear elastic moduli.

\section{Parametrized measure minima} conditions

Our prior analysis has established that under fairly general

$$
E_{\Omega}\left(y_{0}\right)=\inf _{A\left(y_{0}\right)} \int_{\Omega} W(\nabla v) d x=\inf _{A\left(y_{0}\right)} \int_{\Omega} \phi^{* *}(\operatorname{det} \nabla v) d x
$$

Some circumstances under which minimizers actually exist were determined in the preceding section, however, a natural framework from functional analysis from which to seek solutions is not readily available to us owing to the extreme lack of convexity of $w$. The sense in which a minimizing sequence may be said to be compact is rather murky, but two areas of interest may be discerned. These are $H^{1.0}$ and $H^{1.1}$. Analysis in $H^{1.0}$ is not likely to be much different than that in $H^{1 . p}, P>1$, while that in $H^{1.1}$ permits gradients which may tend to measures, suggesting dislocations and other behavior we are not prepared to encounter at this time, cf. $[37,76]$.

Consequently, here we shall consider a parametrized measure minimum associated to a minimizing sequence bounded in $H^{1, \infty}(\Omega)$ and give some thought to equilibrium configurations and their state functions. 
Let $\left(u^{k}\right) \subset A_{\Omega}\left(y_{0}\right)$ be a minimizing sequence which converges in $H^{1, \infty}(\Omega)$ weak* to some $y, y=y_{0}$ on $\partial \Omega$. Here we mean that

$$
\begin{array}{ll}
u^{k} \rightarrow y & \text { in } H^{1, \infty}(\Omega) \text { weak*, } \\
u^{k}=y_{0} & \text { on } \partial \Omega \\
\left\|u^{k}\right\|_{H^{1, \infty}(\Omega)} \leq C, & \text { for all } k, \\
\operatorname{det} F^{k}>0 & \text { in } \Omega, \text { for all } k,
\end{array}
$$

and

$$
E_{\Omega}\left(y_{0}\right)=\lim _{k \rightarrow \infty} \int_{\Omega} w\left(F^{k}\right) d x=\inf _{A\left(y_{0}\right)} \int_{\Omega} \phi^{* *}(\operatorname{det} \nabla v) d x,
$$

where $F^{k}=\nabla u^{k}$. The sequence $\left(u^{k}\right)$ determines a parametrized measure $v=\left(v_{x}\right)_{x \in \Omega}$ with the property

$$
\begin{aligned}
\int_{\Omega} \int_{M} \psi(x, A) \zeta d v_{x} d x & =\lim _{k \rightarrow \infty} \int_{\Omega} \psi\left(x, \nabla u^{k}\right) \zeta d x \\
& \text { for } \psi \in C(\Omega \times M) \text { and } \zeta \in L^{\prime}(\Omega) .
\end{aligned}
$$

Expressed in other words, the weak* limit of the sequence $\left(\psi\left(x, \nabla u^{k}\right)\right)$ is the function

$$
\bar{\psi}(x)=\int_{M} \psi(x, A) d v_{x}, x \in \Omega
$$

Recall that the functions $A, \operatorname{adj} A=\operatorname{det} A A^{-1}$, and $\operatorname{det} A$ are weak* continuous [59]. In particular, for $F=\nabla y$,

$$
\begin{aligned}
F & =\int_{M} A d v_{x}, \\
\operatorname{adj} F & =\int_{M} \operatorname{adj} A d v_{x}, \quad \text { and } \\
\operatorname{det} F & =\int_{M} \operatorname{det} A d v_{x} .
\end{aligned}
$$

It is useful to keep in mind that in the current framework, supp $v$ is compact, indeed, 


$$
\operatorname{supp} \mathcal{C} \bar{\Omega} \times\{|F| \leq C\}
$$

where $C$ is the constant of (5.2). This follows from (5.4) since if $\psi$ is a function with support in the complement of $\bar{\Omega} \times\{|F| \leq C\}$, then

$$
\int_{\Omega} \psi\left(x, \nabla u^{k}\right) \zeta d x=0, \text { for all } k \text {. }
$$

Let us assume throughout this section that (5.1) - (5.5) hold. We begin with some general observations about minima.

Proposition 5.1 Under the assumptions (5.1) - (5.5), y $\in A_{\Omega}\left(y_{0}\right)$ and

$$
E_{\Omega}\left(y_{0}\right)=\int_{\Omega} \phi^{* *}(\operatorname{det} F) d x
$$

Proof: Since $\phi^{* *}$ is convex, it is lower semicontinuous with respect to weak* convergence in $L^{\infty}(\Omega)$. Moreover, as we have noted, the mapping $A \rightarrow \operatorname{det} A$ is weak* continuous, so

$$
\begin{aligned}
\int_{\Omega} \phi^{* *}(\operatorname{det} F) d x & \leq \lim _{k \rightarrow \infty} \int_{\Omega} \phi^{* *}\left(\operatorname{det} F^{k}\right) d x \\
& \leq \lim _{k \rightarrow \infty} \int_{\Omega} W\left(F^{k}\right) d x \\
& =E_{\Omega}\left(y_{0}\right) \\
& =\inf _{A\left(y_{0}\right)} \int_{\Omega} \phi^{* *}(\operatorname{det} \nabla v) d x \\
& \leq \int_{\Omega} \phi^{* *}(\operatorname{det} F) d x .
\end{aligned}
$$

Note in particular that the finiteness of $E_{\Omega}\left(y_{0}\right)$ implies that $y \in A_{\Omega}\left(y_{0}\right)$.

QED

The central technical difficulty met here is that there is no a priori knowledge ensuring that $\operatorname{det}^{k}$ remain bounded away from 0 . This will become even more annoying later when we attempt to decide possible relationships between minimum states and equilibrium states. For the present, we simply observe that the conditions of (5.2) do not 
automatically imply the formulas (5.5) and (5.8) since both $W$ and $\phi^{* *}$ are unbounded as det $F \rightarrow 0$. Nevertheless,

Proposition 5.2 Under the assumptions(5.1)-(5.5), $W$ and $\phi^{* *}$ are $\mathrm{d} v_{\mathrm{x}} \mathrm{dx}$ integrable and

$$
\bar{W}(x)=\bar{\phi}(x)=\bar{\phi}^{* *}(x)=\phi^{* *}(\operatorname{det} F(x)) \text { in } \Omega
$$

Proof. Let $W_{m}(A)=\min \{W(A), m\}$. Thus $W_{m} \geq 0$ and increase to $W$. Now, in view of the preceding Proposition,

$$
\begin{aligned}
\int_{\Omega} \int_{M} W_{m}(A) d v_{x} d x & =\lim _{k \rightarrow \infty} \int_{\Omega} W_{m}\left(F^{k}\right) d x \\
& \leq \lim _{k \rightarrow \infty} \int_{\Omega} W\left(F^{k}\right) d x \\
& =\int_{\Omega} \phi^{* *}(\operatorname{det} F) d x .
\end{aligned}
$$

Thus by the monotone convergence theorem, $W(A)$ is integrable and

$$
\int_{\Omega} \int_{M} W(A) d v_{x} d x \leq \int_{\Omega} \phi^{* *}(\operatorname{det} F) d x
$$

Since $0 \leq \phi^{* *} \leq W, \phi^{* *}$ is also $d v_{x} d x$ integrable. The functions $\bar{\phi}^{* *}$ and $\bar{W}$ may be defined by the formula (5.5) and they are in $L^{\prime}(\Omega)$. By Jensen's inequality and (5.6),

$$
\begin{aligned}
\phi^{* *}(\operatorname{det} F(x)) & =\phi^{* *}\left(\int_{M} \operatorname{det} A d v_{x}\right) \\
& \leq \int_{M} \phi^{* *}(\operatorname{det} A) d v_{x}=\bar{\phi}^{* *}(x) .
\end{aligned}
$$

Thus

$$
\phi^{* *}(\operatorname{det} F) \leq \bar{\phi}^{* *} \leq \bar{W} \quad \text { in } \Omega \text {. }
$$

Combining (5.10) and (5.11),

$$
\int_{\Omega} \phi^{* *}(\operatorname{det} F) d x \leq \int_{\Omega} \bar{\phi}^{* *} d x \leq \int_{\Omega} \bar{W} d x \leq \int_{\Omega} \phi^{* *}(\operatorname{det} F) d x,
$$


so equality holds, and by (5.11),

$$
\bar{W}=\bar{\phi}^{* *}=\phi^{* *}(\operatorname{det} F) \text {. }
$$

In view of the pointwise inequality $\phi^{* *} \leq \phi \leq W$, it follows also that $\bar{W}(x)$ $=\bar{\phi}(x)=\bar{\phi}^{* *}(x)$.

QED

We proceed to begin a direct analysis of the parametrized measure at a minimum. To complete this analysis we shall be obliged to discuss equilibrium conditions.

Lemma 5.3 Let $\left(v_{\mathrm{x}}\right)_{\mathrm{x} \in \Omega}$ be a parametrized measure minimum. Then

$\operatorname{supp} v_{\mathrm{x}} \subset\left\{\mathrm{A}: \mathrm{a} \leq \operatorname{det} \mathrm{A} \leq \mathrm{D}, \phi^{* *}\right.$ is affine in $[\mathrm{a}, \mathrm{b}]$, anddet $\left.\mathrm{F}(\mathrm{x}) \in[\mathrm{a}, \mathrm{b}]\right\}$

almost everywhere in $\Omega$. In particular, if $\phi$ is strictly convex, then

$$
\operatorname{supp} v_{x} \subset\{A: \operatorname{det} A=\operatorname{det} F(x)\} \text { a.e. } \operatorname{in} \Omega \text {. }
$$

Proof. Fix $x \in \Omega, a$ and $b$ such that $\operatorname{det} F(x) \in[a, b], \phi^{* *}$ is affine on $[a, b]$, and set $v=v_{x}$. It is possible here that $b=+\infty$; the argument is valid in this case as well. So there are numbers $\alpha$ and $\beta$ such that

$$
\begin{array}{lll}
\phi^{* *}(\mathrm{t})-\alpha \mathrm{t}-\beta \geq 0 & \text { in } & (0, \infty), \\
\phi^{* *}(\mathrm{t})-\alpha \mathrm{t}-\beta=0 & \text { in } & {[\mathrm{a}, \mathrm{b}], \text { and }} \\
\phi^{* *}(\mathrm{t})-\alpha \mathrm{t}-\beta>0 & \text { in } & (0, \infty)-[\mathrm{a}, \mathrm{b}] .
\end{array}
$$

Since $\operatorname{det} F(x) \in[a, b]$, by the preceding proposition,

$$
\begin{aligned}
& 0=\phi^{* *}(\operatorname{det} F(x))-\alpha \operatorname{det} F(x)-\beta \\
& =\int_{M}(\phi * *(\operatorname{det} A)-\alpha \operatorname{det} A-\beta) d v \\
& =\int_{\text {supp } v}\left(\phi^{* *}(\operatorname{det} A)-\alpha \operatorname{det} A-\beta\right) d v .
\end{aligned}
$$

Hence

$$
\phi^{* *}(\operatorname{det} A)=\alpha \operatorname{det} A-\beta \quad \text { a.e. on } \operatorname{supp} v \text {. }
$$


This is our principal result about the support of the parametrized measure. Some discussion of it follows the proof.

Theorem 5.4 Let $\left(v_{\mathrm{x}}\right)_{\mathrm{x} \in \Omega}$ be a parametrized measure minimum. Then

$$
W(A)=\phi(\operatorname{det} A)=\phi^{* *}(\operatorname{det} A) \text { a.e. insupp } v_{x}, \text { z.e. in } \Omega \text {, }
$$

and in particular:

$\operatorname{supp} v_{x} \subset\left\{A^{\prime}: a \leq \operatorname{det} A \leq b, \phi^{* *}\right.$ is affine in $[a, b], \operatorname{det} F(x) \in[a, b]$, and $\left.\phi^{* *}(\operatorname{det} A)=W(A)\right\}$.

Proof. Since $W(A)-\phi^{* *}(\operatorname{det} A) \geq 0, A \in \mathbb{M}$, and

$$
\int_{M}\left(W(A)-\phi^{* *}(\operatorname{det} A)\right) d v=\bar{W}(x)-\phi^{* *}(\operatorname{det} F(x))=0,
$$

it is immediate that

$$
W(A)=\phi^{* *}(\operatorname{det} A) \text { on } \operatorname{supp} v .
$$

QED

The import of this last result is that the variational principle does not permit the oscillating nature of a minimizing sequence to introduce any phantom states which are not realizable by the original energy density. For example, the parametrized measure which describes an austenite/ martensite transition may represent a mixture of the two phases, but its support contains no intermediate phases which, at least in theory, are not pointwise attainable by the energy density $W$. 


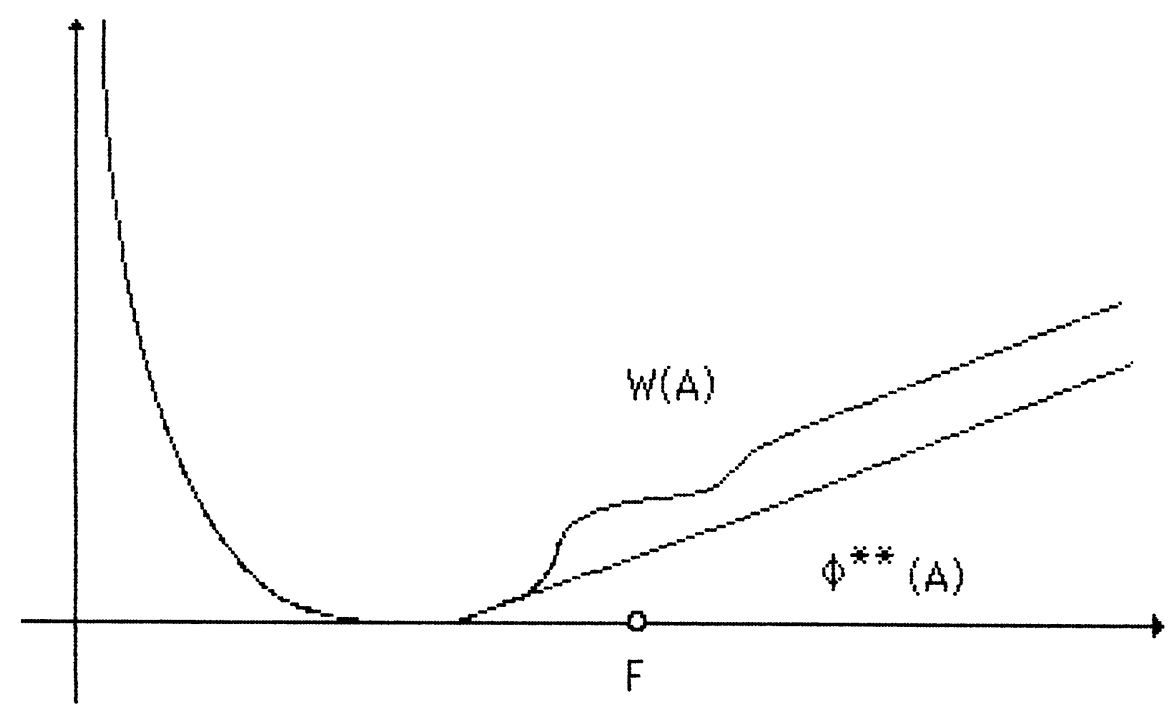

An energy density $W$ with an asymptotic direction.

There can be no parametrized measure associated to the indicated $F$.

Corollary $5.5 \quad$ Let $\left(v_{x}\right)_{x \in \Omega}$ be a parametrized measure minimum. Then the Piola-Kirchhoff stress is given by

$$
\bar{S}=-\tau \operatorname{det} F F^{-\top}, \tau=-\phi^{* *}(\operatorname{det} F), \text { in } \Omega
$$

We are not claiming at this moment that $\bar{s}$ has any mechanical meaning, but only that the formula (5.13) holds.

Proof. Recall that $S=\partial W / \partial A$. We assume here, as usual, that $W$ is smooth. The function $W(A)-\phi^{* *}(\operatorname{det} A)$ attains its minimum value 0 on $\operatorname{supp} v$, so

$$
S(A)=\phi^{* *}(\operatorname{det} A) \operatorname{det} A A^{-\top} \text { on } \operatorname{supp} v_{x} .
$$

Now $\phi^{* *}$ is linear on $\operatorname{supp} v_{x}$ thus $\phi^{* *}$ is constant there, by the theorem. Hence,

$$
S(A)=\phi * *(\operatorname{det} F(x)) \operatorname{det} A A^{-T} \text { on } \operatorname{supp} v_{x} \text {. }
$$

Integrating this gives that

$$
\bar{S}(x)=\int_{M} S(A) d v
$$




$$
=\phi^{* *}(\operatorname{det} F(x)) \int_{M} \operatorname{det} A A^{-T} d v_{x} .
$$

Recall that $\operatorname{det} A A^{-1}=\operatorname{adj} A$ is weakly continuous, cf. (5.6), whence

$$
\int_{M} \operatorname{det} A A^{-T} d v_{x}=\operatorname{det} F F^{-T}
$$

which proves (5.13).

QED

Another consequence of the theorem is

Corollary $5.6 \quad$ Let $\phi$ be strictly convex. Then, with the notations of (5.1)-(5.5), then for a subsequence of $k$,

$$
\operatorname{det} \mathrm{F}^{\mathrm{k}} \rightarrow \operatorname{det} \mathrm{F} \quad \text { in } \mathrm{L}^{\mathrm{P}}(\Omega), \quad 1 \leq \mathrm{p}<\infty \text { and a.e. in } \Omega \text {. }
$$

Proof. This is standard, since $v$ reduces to a delta function of functions of the determinant according to the theorem.

QED

\section{Stable parametrized measure minima}

Most desirably, an equilibrium solution renders stationary the energy functional within a suitable class of disturbances. This is an attribute of stability which is not necessarily conferred on a state which realizes minimum energy. Much has been written about this, especially by Ball [2], [3], and it is fair to report that our difficulties here are connected to the growth of $W$ as detF $\rightarrow 0$. For example, that $\left(v_{x}\right)_{x \in \Omega}$ is a parametrized measure minimum ought to mean that

$$
\int_{\Omega} \bar{W} d x \leq \int_{\Omega} \int_{M} W(A+\nabla \zeta) d v_{x} d x, \zeta \in H_{0}^{1} \cdot \mathbf{w}(\Omega),
$$

at least for $\|\zeta\|_{H^{1, \infty}}(\Omega)$ sufficiently small. Unfortunately, since nothing is established about detF, it is not evident that there are any $\zeta$ for which the right hand side is finite.

Conditions for variation of domain are the most useful to us, so our criteria for stability will be expressed in these terms. The simplest condition, although it is stronger than necessary, may be formulated by 
first discussing the situation for a Lipschitz minimum.

Suppose that $u(x)$ is a Lipschitz minimum and consider the variations

$$
u_{\epsilon}(x)=u(x+\epsilon \zeta(x)) \quad \text { for } \zeta \epsilon H_{0}^{1,0}(\Omega), \epsilon>0 .
$$

Then

$$
\int_{\Omega} W\left(\nabla u_{\epsilon}\right) d x=\int_{\Omega} W(\nabla u(x+\epsilon \zeta)(1+\epsilon \nabla \zeta)) d x
$$

When the right hand side of (6.2) is finite for $\|\zeta\|_{H^{1, \infty}}(n)$ suitably small, we would say that $u$ is an admissible solution. It would be a local minimum provided that

$$
\int_{\Omega} w(\nabla u) d x \leq \int_{\Omega} w\left(\nabla u_{\epsilon}\right) d x
$$

for $\zeta$ suitably restricted. Changing variables, $z=x+\epsilon \zeta(x)$, (6.2) may be rewritten

$$
\int_{\Omega} w\left(\nabla u_{\epsilon}\right) d x=\int_{\Omega} w(\nabla u(1+\epsilon \nabla \zeta)) \operatorname{det}(1+\epsilon \nabla \zeta)^{-1} d z
$$

To extend this notion to a parametrized measure, we say that $\left(v_{x}\right)_{x \in \Omega}$ is a stable equilibrium solution if there exists a sequence $\left(u^{k}\right)$ and $y \in H^{1 . \infty}(\Omega)$ such that

$$
\begin{aligned}
& u^{k} \rightarrow y \quad \text { in } H^{1, \infty}(\Omega) \text { weak*, } y=y_{0} \text { on } \partial \Omega, \\
& \operatorname{det}^{k}>0, F^{k}=\nabla u^{k} \text { for each } k \\
& \left(u^{k}\right) \text { determine the parametrized measure }\left(v_{x}\right)_{x \in \Omega}
\end{aligned}
$$

with

$$
\int_{n} \bar{W} d x<\infty
$$

and, for each $\zeta \in H_{0}^{1} 0^{\infty}(\Omega)$, there is an $\epsilon_{0}>0$ such that

$$
\int_{\Omega} \bar{W} d x \leq \int_{\Omega} \int_{M} W(A(1+\epsilon \nabla \zeta)) \operatorname{det}(1+\epsilon \nabla \zeta)^{-1} d v_{x} d x<\infty,
$$


for $\epsilon<\epsilon_{0}$.

The properties (6.4) have the automatic consequences that

$$
F=\nabla y=\int_{\Omega} A d v_{x} \text { and } \operatorname{det} F=\int_{\Omega} \operatorname{det} A d v_{x} \text { a.e. in } \Omega \text {. }
$$

The condition (6.5) implies a familiar version of the Euler-Lagrange equations,

$$
\int_{\Omega} \int_{M}\left\{A^{\top} S(A) \cdot \nabla \zeta-W(A) \operatorname{div} \zeta\right\} d v_{x} d x=0, \zeta \in H_{0}^{1} \cdot \text { o }(\Omega) \text {. (6.6) }
$$

The stability portion of (6.5) is the insistence that the right hand side be finite. This may be relaxed. For example, given $\zeta \in H_{0}^{1},{ }^{\circ}(B), B=\{|z|<$ 1\}, $\xi \in \Omega$, let $\zeta_{\epsilon}=\epsilon \zeta((x-\xi) / \epsilon)$ and integrate with respect to $\xi$ in (6.5). Set $z=(x-\xi) / \epsilon$. A relaxed condition for admissibility is that

$$
\int_{\Omega} \int_{B} \int_{M} W(A(1+\nabla \zeta(z))) d v_{x} d z d x<\infty .
$$

We shall take up this and related conditions in the next section.

Observe that if

$$
\operatorname{det} F>c>0 \text { in } \Omega \text {, }
$$

then $\left(v_{x}\right)_{x \in \Omega}$ is an admissible solution in the sense if (6.5), (6.6).

Also note that if

$$
m(\operatorname{det} A)^{-\mu} \leq W(A) \leq M(\operatorname{det} A)^{-\mu}+C
$$

for some $0<m<M, \mu>0$, and $C \geq 0$, then any parametrized measure obeying (6.4) satisfies (6.5). With the addition of a hydrostatic pressure, the densities considered by Eftis, MacDonald, and Arkilic [17,18] satisfy (6.9)

Theorem 6.1 Assume that $\left(v_{\mathrm{x}}\right)_{\mathrm{x \in} \Omega}$ is a parametrized measure minimum if the sense of (5.1)-(5.5) which is stable in the sense of (6.4) and(6.5). Then 


$$
\tau=-\phi * *^{\prime}(\operatorname{det} F)
$$

is constant in $\Omega$. In the case that $\phi$ is strictly convex, then det $F$ is also constant in $\Omega$.

Thus the Cauchy stress of the deformed configuration,

$$
T=-\tau 1
$$

reduces to a constant pressure.

Proof. We may avail ourselves of the equilibrium equation (6.6). We evaluate the various terms it contains. Note that

$$
A^{\top} S(A)=\phi^{* *}(\operatorname{det} A) \operatorname{det} A 1 \text { on } \operatorname{supp} v_{x} \text {. }
$$

so by Theorem 5.4 and the weak* continuity of $\operatorname{det} A$,

$$
\int_{M} A^{\top} S(A) d v_{x}=\phi^{* *}(\operatorname{det} F) \operatorname{det} F 1 \text { in } \Omega \text {. }
$$

Also we know that

$$
\int_{M} W(A) d v_{x}=\phi^{* *}(\operatorname{det} F) \quad \text { in } \Omega
$$

Thus (6.6) becomes

$$
\int_{\Omega}\left\{\phi^{* *}(\operatorname{det} F) \operatorname{det} F 1-\phi * *(\operatorname{det} F) 1\right\} \cdot \nabla \zeta d x=0, \quad \zeta \in H_{0}^{1}{ }^{\infty}(\Omega) .
$$

From this we infer that

$$
\phi^{* *}(\operatorname{det} F) \operatorname{det} F-\phi^{* *}(\operatorname{det} F)=c=\text { const. in } \Omega \text {. }
$$

Given two points $x_{1}, x_{2} \in \Omega$, let $F_{1}=F\left(x_{1}\right)$, so the above, (6.11), tells us that

$$
\phi^{* *}\left(\operatorname{det} F_{1}\right) \operatorname{det} F_{1}-\phi^{* *}\left(\operatorname{det} F_{1}\right)=\phi^{* *}\left(\operatorname{det} F_{2}\right) \operatorname{det} F_{2}-\phi * *\left(\operatorname{det} F_{2}\right) .
$$


By the convexity of $\phi^{* *}$, we also know that

$$
\phi^{* *}\left(\operatorname{det} F_{2}\right)-\phi^{* *}\left(\operatorname{det} F_{1}\right) \geq \phi^{* *}\left(\operatorname{det} F_{1}\right)\left(\operatorname{det} F_{2}-\operatorname{det} F_{1}\right) \text {. }
$$

Combining these we deduce that

$$
\phi^{* *}\left(\operatorname{det} F_{2}\right) \geq \phi^{* *}\left(\operatorname{det} F_{1}\right)
$$

Since the roles of $F_{1}$ and $F_{2}$ may be interchanged, (6.10) follows.

In the case where $\phi$ is strictly convex, $\phi^{\prime}$ is $1: 1$, so $\operatorname{det} F$ is also constant in $\Omega$.

\section{Parametrized measure equilibria}

As an alternative approach to the direct analysis of the measure, we direct our attention to the behavior of the energy density $W$ at an equilibrium configuration. This will not require that the Young measure give an absolute minimum of energy, and our conclusions will reflect this. While dynamical considerations may offer the most satisfactory notions of metastability, as developed for example in Andrews and Ball [1] and Pego [62],[63], these are not yet available to us for our three dimensional problem.

In order to confine ourselves to the essential features of the analysis, we shall consider parametrized measures which minimize energy with respect to small compactly supported perturbations. Under these circumstances, we shall deduce a form of quasiconvexity. Similar considerations in a different context were initiated independently by $\mathrm{J}$. Ball [5].

Let $\Omega \subset \mathbb{R}^{3}$ be a bounded domain with Lipschitz boundary and $\left(v_{x}\right)_{x \in \Omega}$ be a parametrized measure in the sense that there is a sequence $\left(u^{k}\right.$ ) and $y$ in $H^{1, \infty}(\Omega)$ such that

$$
\begin{aligned}
& u^{k} \rightarrow y \quad \text { in } H^{1, \infty}(\Omega) \text { weak* } \\
& \operatorname{det}^{k}>0 \text { in } \Omega, \quad F^{k}=\nabla u^{k},
\end{aligned}
$$

and $\left(u^{k}\right)$ determine the parametrized measure $\left(v_{x}\right)_{x \in \Omega}$. Suppose also that 


$$
\bar{W}(x)=\int_{M} W(A) d v_{x}
$$

satisfies

$$
\int_{\Omega} \bar{W}(x) d x<\infty
$$

As before, $F=\nabla y$ is represented by the formula

$$
F(x)=\int_{M} A d v_{x}
$$

Again we shall be concerned with variations of domain. In order to motivate our point of view, it may be helpful to begin with a local

minimum $y(x)$ assumed to be Lipschitz with $F=\nabla y$ satisfying det $F \geq c$ $>0$. For $\zeta \in H_{0}^{1} \cdot{ }^{\circ}(D)$, where $D$ is a fixed bounded open set, with sufficiently small gradient and $x_{0} \in \Omega$ fixed, consider the variation

$$
\begin{array}{ll}
y_{\epsilon}(x)=y\left(x+\epsilon \zeta\left(\left(x-x_{0}\right) / \epsilon\right)\right) & x \in x_{0}+\epsilon D \\
y_{\epsilon}(x)=y(x) & x \in \Omega \backslash\left(x_{0}+\epsilon D\right), \epsilon>0 .
\end{array}
$$

So

$$
\nabla y_{\epsilon}(x)=F\left(x+\epsilon \zeta\left(\left(x-x_{0}\right) / \epsilon\right)\right)\left(1+\nabla \zeta\left(\left(x-x_{0}\right) / \epsilon\right)\right)
$$

and

$$
\operatorname{det} \nabla y_{\epsilon}(x)=\operatorname{det} F\left(x+\epsilon \zeta\left(\left(x-x_{0}\right) / \epsilon\right)\right) \operatorname{det}\left(1+\nabla \zeta\left(\left(x-x_{0}\right) / \epsilon\right)\right) \geq \frac{1}{2} c
$$

say, provided $|\nabla \zeta|$ is small enough. Thus

$$
\int_{\Omega} W\left(\nabla y_{\epsilon}\right) d x<\infty
$$

If we set $z=\left(x-x_{0}\right) / \epsilon$ in this integral we obtain

$$
\int_{D} W\left(F\left(x_{0}+\epsilon z+\epsilon \zeta(z)\right)(1+\nabla \zeta(z))\right) d z<\infty
$$

We may require finiteness of this integral as a condition on the variation 
$\zeta$. However we prefer to assume that it is finite only for most choices of $x_{0}$ in the sense that

$$
\int_{\Omega^{\prime}} \int_{D} W\left(F\left(x_{0}+\epsilon Z+\epsilon \zeta(z)\right)(1+\nabla \zeta(z))\right) d z d x_{0}<\infty
$$

for $\Omega^{\prime} c \subset \Omega$ and $\epsilon$ small. Interchanging the $d x_{0} d z$ integrals and changing variables, $x=x_{0}+\epsilon z+\epsilon \zeta(z)$, this becomes simply

$$
\int_{D} \int_{\Omega} \cdot W(F(x)(1+\nabla \zeta(z)) d x d z<\infty .
$$

Thus we shall adopt as our admissibility criterion for $\left(v_{x}\right)_{x \in \Omega}$ that

$$
\int_{\Omega} \int_{D} \int_{M} W(A(1+\nabla \zeta(z))) d v_{x} d z d x<\infty
$$

whenever $\zeta \in H_{0}^{1 . \infty}(D)$ and $|\nabla \zeta|$ is sufficiently small.

With this in mind, we say that $\left(v_{x}\right)_{x \in \Omega}$ is an equilibrium measure with the underlying deformation $y$ provided that whenever (7.4) holds

$$
\int_{D} \bar{W}(x+\epsilon z) d z \leq \int_{D} \int_{M} W(A(1+\nabla \zeta(z))) d v_{x+\epsilon z+\epsilon \zeta(z)} d z
$$

for $x \in \Omega$ a.e.

We begin by illustrating a property of quasiconvexity of $W$ at an equilibrium position.

Theorem 7.1 Let $\left(v_{\mathrm{x}}\right)_{\mathrm{x \in \Omega}}$ be an admissible equilibrium in the sense of (7.1) - (7.5). Then

$$
\bar{W}(x) \leq|D|^{-1} \int_{D} \int_{M} W(A(1+\nabla \zeta(z))) d v_{x} d z \quad \text { for } x \in \Omega \text {. (7.6) }
$$

Proof. The object is to justify allowing $\epsilon$ to pass to 0 in (7.5). Let $\Omega$ ' $c \subset \Omega$ and $\epsilon>0$ be small and set

$$
g(z)=\int_{\Omega} \int_{M} W(A(1+\nabla \zeta(z))) d v_{x} d x \in L^{\prime}(D)
$$


and

$$
g_{\epsilon}(z)=\int_{\Omega^{*}} \int_{M^{\prime}} W(A(1+\nabla \zeta(z))) d v_{x+\epsilon z+\epsilon \zeta(z)} d x \leq g(z)
$$

Since $g(z) \in L^{\prime}(\Omega)$, the function

$$
W(x, z)=\int_{M} W(A(1+\nabla \zeta(z))) d v_{x} \in L^{\prime}(\Omega \times D) .
$$

Thus, since translation is continuous in $L$,

$$
h_{\varepsilon}(z)=\int_{D}|w(x+\epsilon z+\epsilon \zeta(z), z)-w(x, z)| d x \rightarrow 0 \text { as } \epsilon \rightarrow 0 \text {, a.e. } z \in D \text {, }
$$

and also

$$
h_{\epsilon}(z) \leq g_{\epsilon}(z)+g(z) \leq 2 g(z)
$$

By Lebesgue's Theorem we infer that

$$
\lim _{\epsilon \rightarrow 0} \int_{\Omega} h_{\epsilon}(z) d z=0
$$

This means that

$$
\begin{array}{r}
\lim _{\epsilon \rightarrow 0} \int_{D} \int_{\Omega^{\prime}} l \int_{M} W(A(1+\nabla \zeta(z))) d v_{x+\epsilon z+\epsilon \zeta}(z) \\
-\int_{M} W(A(1+\nabla \zeta(z))) d v_{x} \mid d x d z \\
=0
\end{array}
$$

Here we may intechange the $x$ and $z$ integrals, whence

$$
\begin{array}{r}
\lim _{\epsilon \rightarrow 0} \int_{\Omega^{\prime}} \int_{D}\left|\int_{M} W(A(1+\nabla \zeta(z))) d v_{x+\epsilon z+\epsilon \zeta(z)}-\int_{M} W(A(1+\nabla \zeta(z))) d v_{x}\right| d z d x \\
=0
\end{array}
$$

and therefore

$$
\lim _{\epsilon \rightarrow 0} \int_{D} \| \int_{M} W(A(1+\nabla \zeta(z))) d v_{x+\epsilon z+\epsilon \zeta(z)}-\int_{M} W(A(1+\nabla \zeta(z))) d v_{x} \mid d z=0
$$

Since $\bar{W}(x) \in L^{1}(\Omega)$, almost every $x$ is a density point of $\bar{W}$, so 


$$
\bar{W}(x)=\lim _{\epsilon \rightarrow 0}|D|^{-1} \int_{D} \bar{W}(x+\epsilon z) d z
$$

Finally, using the minimizing property (7.5) and (7.7) above,

$$
\begin{aligned}
\bar{W}(x) & =\lim _{\epsilon \rightarrow 0}|D|^{-1} \int_{D} \bar{W}(x+\epsilon Z) d z \\
& \leq \lim _{\epsilon \rightarrow 0}|D|^{-1} \int_{D} \int_{M} W(A(1+\nabla \zeta(z))) d v_{x+\epsilon z+\epsilon \zeta(z)} d z \\
& =|D|^{-1} \int_{D} \int_{M} W(A(1+\nabla \zeta(z))) d v_{x} d z .
\end{aligned}
$$

A consequence of this theorem is the rank-one convexity of $W$ at an equilibrium solution. It may help to recall that $\operatorname{supp} v_{x}$ is compact for each $x \in \Omega$.

Corollary 7.2 Let $\left(v_{\mathrm{x}}\right)_{\mathrm{x \in \Omega}}$ be an admissible equilibrium in the sense of (7.1) - (7.5). Then

$$
\bar{W}(x) \leq \int_{M}\left\{\lambda W(A(1+a \otimes b))+(1-\lambda) W\left(A\left(1+\left(\lambda / 1_{-\lambda}\right) a \otimes b\right)\right)\right\} d v_{x}
$$

a.e. in $\Omega$ whenever $W(\mathrm{~A}(1+\mathrm{a} \otimes \mathrm{b}))$ and $\mathrm{W}(\mathrm{A}(1+(\lambda / 1-\lambda) \mathrm{a} \otimes \mathrm{b}))$ are boundec on $\operatorname{supp} v_{\mathrm{x}}, 0 \leq \lambda<1$, and $|\mathrm{a}|,|\mathrm{b}|$ are sufficiently small.

Proof. This is a variant of a well known argument. Choose $D=\mathbb{Q}$, a cube of given side length and suppose $|D|=1$. Let $x$ be the characteristic function of a real interval with the property that

$$
|\mathbb{Q}|^{-1} \int_{0} x(b \cdot x) d z=\lambda, \quad(z=D \cdot x)
$$

so that $x^{k}(z)=x(k b \cdot x), k=1,2,3, \ldots$ satisfy

$$
x^{k} \rightarrow \lambda \text { in } L^{\infty}(\mathbb{Q}) \text { weak* as } k \rightarrow \infty
$$

Determine $v^{k}$ by 


$$
\nabla v^{k}=x^{k} a \otimes b-\left(1-x^{k}\right)(\lambda / 1-\lambda) a \otimes b,
$$

so that

$$
\begin{aligned}
& v^{k} \rightarrow 0 \text { uniformly in } \mathbb{Q} \text { and } \\
& \nabla v^{k} \rightarrow 0 \text { in } L^{\infty}(\mathbb{Q}) \text { weak* as } k \rightarrow \infty \text {. }
\end{aligned}
$$

Set $\zeta^{k}=\eta v^{k}$, where $\eta=0$ near $\partial Q$. Hence the $\zeta^{k}$ also have the property that

$$
\zeta^{k} \rightarrow 0 \text { in } L^{\infty}(\mathbb{Q}) \text { weak* as } k \rightarrow \infty
$$

and they vanish on $\partial \mathbb{Q}$ as well. Assume that $a, b$ are small enough that (7.4) and (7.5) hold for $\zeta^{k}$. By (7.6),

$$
\bar{W}(x) \leq|\mathbb{Q}|^{-1} \int_{\mathbb{Q}} \int_{M} W\left(A\left(1+\nabla \zeta^{k}(z)\right)\right) d v_{x} d z
$$

Note that

$$
g^{k}(A)=|\mathbb{Q}|^{-1} \int_{Q} W\left(A\left(1+\nabla \zeta^{k}(z)\right)\right) d z
$$

are bounded on $\operatorname{supp} v_{\mathrm{x}}$ for $k$ sufficiently large by our hypothesis and converge in $L^{\infty}(\mathbb{M})$ weak* to

$$
g(A)=|\mathbb{Q}|^{-1} \int_{\mathbb{Q}}\{\lambda W(A(1+a \otimes D \eta))+(1-\lambda) W(A(1+(\lambda / 1-\lambda) a \otimes b \eta))\} d z .
$$

In particular,

$$
\bar{W}(x) \leq \int_{M} g(A) d v_{x} .
$$

The result follows by letting $\eta \rightarrow 1$ in $\mathbb{Q}$.

Differentiating (7.8) with respect to $\lambda$ at $\lambda=0$ gives that

$$
\int_{M} W(A(1+a \otimes b)) d v_{x}-\int_{M} W(A) d v_{x}-\int_{M} A^{\top} S(A) \cdot a \otimes b d v_{x} \geq 0 .
$$

If the condition on the smallness of $|\nabla \zeta|$ in (7.4) is generous enough to 
permit $a \otimes b=L e_{1} \otimes L^{-\top} e_{j}$ and $a \otimes b=L\left(e_{i}+e_{j}\right) \otimes L^{-\top}\left(e_{j}-e_{j}\right)$ then $1+a \otimes b \in H$, $W(A(1+a \otimes b))=W(A)$, and it follows that

$$
\int_{M} A^{\top} S(A) d v_{x}=-\tau(x) 1
$$

So as soon as the size of the perturbations is allowed to exceed the size of the lattice spacing, the present formulation predicts that the Cauchy stress is a pressure. This is one conceivable approach to the question of metastability. It may be of value to remember that (7.10) may hold in metastable states which are not absolute minima, for example in an austenitic phase where martensitic phases give lowest energy. On the other hand, it would appear that there are many circumstances where the energy barrier between lattice invariant shear transformations may be much greater than that between closely related phases within, roughly speaking, the same energy well. Some of James' ideas about energy densities are based on this observation [38-43].

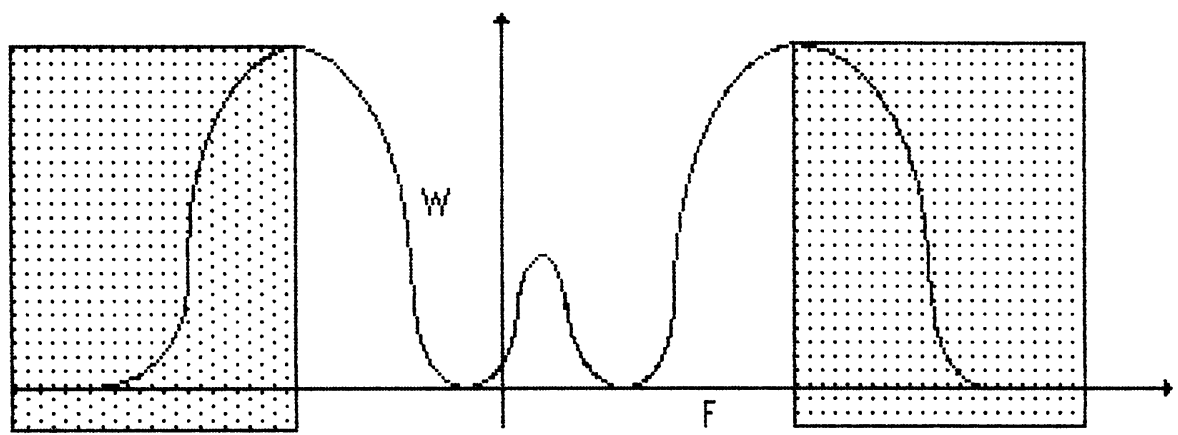

The energy barrier between lattice invariant shearsmay be much greater than the barriers within a well, suggesting a restricted energy density, cf. James [41].

With Theorem 7.1 in mind, we may define a parametrized measure $\mu$ $=\left(v_{x}\right)_{z \in D}$ for a fixed $x \in \Omega$, that is $\mu_{z}=v_{x}$ for each $z \in D$. Such $a$ measure $\mu$ corresponds to the homogeneous deformation $F(x) z$. Is it admissible?

Theorem 7.3 Let $\left(v_{x}\right)_{x \in \Omega}$ be an admissible equilibrium measure in the sense of $(7.1)-(7.5)$. Then $\left(\mu_{z}\right)_{z \in D}$, where $\mu_{z}=v_{x}$ for each $z \in \mathrm{D}$, is an admissible equilibrium measure for almost every $x \in \Omega$.

Proof. We need only show that $\mu$ is admissible since the equilibrium property (7.5) follows by applying Theorem 7.1 to $v$. To show this, we 
have to provide a sequence whose parametrized measure is $v_{x_{0}}$ for almost every $x_{0} \in \Omega$. We may assume that $D=\mathbb{Q}$, a unit cube centered at 0 .

Let $x_{0} \in \Omega$ and $\rho>0$. Set

so

$$
v^{k}(z)=\rho^{-1}\left(u^{k}\left(x_{0}+\rho z\right)-u^{k}\left(x_{0}\right)\right), \quad z \in \mathbb{Q},
$$

$$
\nabla v^{k}(z)=\nabla u^{k}\left(x_{0}+\rho z\right)=F^{k}\left(x_{0}+\rho z\right) \text {. }
$$

For any continuous function $\psi(A)$,

$$
\int_{Q} \psi\left(\nabla v^{k}\right) d z=\rho^{-n} \int_{Q\left(x_{0}, \rho\right)} \psi\left(F^{k}\right) d x \text {. }
$$

where $n=2$ or 3 and $\mathbb{Q}\left(x_{0}, \varrho\right)=x_{0}+\rho \mathbb{Q}$. We write this as

$$
\int_{Q} \psi\left(\nabla v^{k}\right) d z=\int_{Q} \psi\left(F^{k}\right) \zeta_{p} d x
$$

where $\zeta_{p}=p^{-n} x_{\mathbb{Q}\left(x_{0}, p\right)} \in L^{\prime}(\mathbb{Q})$. Now $\left\|\zeta_{p}\right\|_{L^{\prime}(\mathbb{Q})}=|\mathbb{Q}|=1$ and

$$
\zeta_{p} \rightarrow \delta_{x_{0}} \quad \text { in } M(\mathbb{Q}) \text { weak*, }
$$

that is, in the sense of measures.

Let $\epsilon>0$. Then we can find a $k(\rho, \epsilon)$ such that

$$
\left|\int_{Q} \psi\left(F^{k}\right) \zeta_{p} d x-\int_{Q} \int_{M} \psi(A) \zeta_{p} d v_{x} d x\right|\langle\epsilon \text { for } k>k(\rho, \epsilon) \text {. }
$$

Suppose that $x_{0}$ is a Lebesgue point of the function

$$
\psi(x)=\int_{M} \psi(A) d v_{x} \in L^{\infty}(\mathbb{Q})
$$

Then for $\rho$ sufficiently small

$$
\left|\int_{0} \int_{M} \psi(A) \zeta_{p} d v_{x} d x-\bar{\psi}\left(x_{0}\right)\right|<\epsilon .
$$

Consequently we may extract a sequence $\rho_{j} \rightarrow 0$ and $k_{j} \rightarrow \infty$ such that 


$$
w^{j}(z)=e_{j}^{-1}\left(u^{k_{j}}\left(x_{0}+e_{j} z\right)-u^{k_{j}}\left(x_{0}\right)\right)
$$

has the property

$$
\lim _{j \rightarrow \infty} \int_{Q} \psi\left(\nabla w^{j}\right) d z=\int_{M} \psi(A) d v_{x_{0}} .
$$

It follows, since $C(M)$ is separable, that we may find a subsequence of the $\left(w^{j}\right)$ such that (7.11) holds for all $\psi \in C(M)$.

QED

\section{Some concluding remarks}

When an energy density does not give rise to a lower semicontinuous functional, it is a formidable question to identify its minimizers and minima. In the case when the boundary conditions are homogeneous, this is equivalent to identifying the function

$$
\tilde{W}(F)=E_{\Omega}(F) /|\Omega|=\inf _{A(F)}|\Omega|^{-1} \int_{\Omega} W(\nabla V) d x .
$$

The first part of this work was devoted to establishing that

$$
\tilde{W}(F)=\phi^{* *}(\operatorname{det} F)
$$

the convexification of the subenergy of $W$ introduced by Ericksen [24]. The argument exploited the symmetries of $W$ but was hampered by the constraints on the kinematic compatibility and the determinants of the admissible functions. In abstract, the function $\phi^{* *}$ (detF) may be viewed as the analogue of the effective modulus of concern in the homogenization of periodic structures $[10,49]$. Note that it is polyconvex. The notion of quasiconvexity itself was introduced by Morrey, cf. [59], and some interesting work is due to Ball [2] and Meyers [57].

Explicit computation of $\tilde{W}$ is not often found in the literature. Another important case where it has been computed is in the optimal design questions considered by Kohn and Strang [50,51,52]. Their papers explain much of the origins of these ideas. Smoothness in this case has been established in [12]. Pipkin $[64,65]$ has considered another situation where it is important to compute $\tilde{W}$. 
The device adopted to show (8.2) consists in choosing a sequence of deformations with highly oscillatory gradients, which is much in the style of the homogenization of periodic structures [58], but differs in several notable respects. The constraints on the admissible deformations we mentioned above are one difference. Another is that the material itself chooses the scale of and proportion of the phases which are to be averaged. This is not merely a mathematical artifact, but is inherent in the lattice constants and the energy of the material. Ball and James [6] give excellant illustrations of this.

Many alloys and ionic solids undergo phase transformations from a more symmetric (austenitic) phase to less symmetric (martensitic) ones as their thermal environment is changed. Frequently the less symmetric phase is susceptible to twinned configurations, but these are rarely compatible with the more symmetric phase, if ever. For an introduction to the kinematics of twinning, the mathematical reader is referred to [31]. At critical temperature, twinned phases of martensite tend to average themselves in order that the average deformation achieve kinematic compatibility with the austenite across the phase front. This is called fine phase twinning by Ball and James [6].

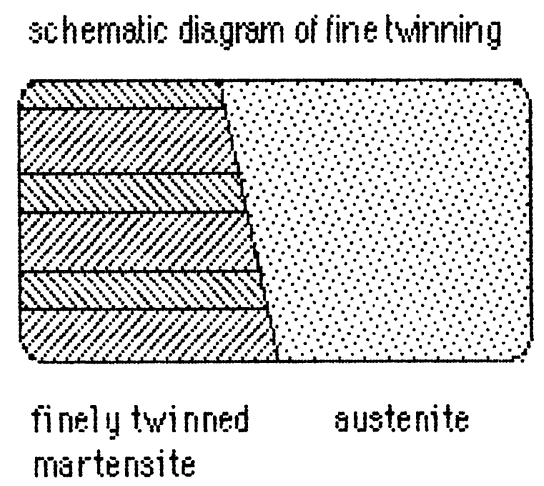

Simple examples of this visible in the optical microscope are the cubic to tetragonal transitions in indium thallium and barium titanate $[9,11,44]$. Many other examples are known, cf. Barrett and Massalski [8]. of historical interest, zinc blende is a face centered cubic crystal often found in a striated form which is stable over a wide temperature range [73]. This may be an example of fine twinning in the strict crystallographic sense or an example of a fine scale structure derived from the presence of many stacking faults in the twin planes [8]. For our purposes, this is only a question of scale in as much as the kinematic and 
energetic description of the macroscopic deformation is the same under either hypothesis.

The authors would like to express their appreciation to J. L. Ericksen for his continuous assistance and interest in this project. They would also like to thank J. Ball, I. Fonseca, and R. James for their many useful discussions.

This work was begun while the authors were in residence at the IMA at the University of Minnesota during the program Continuum Physics and Partial Differential Equations, 1984-1985. Research partially supported by NSF grants MCS 83-01375 and DMS 87-0672.

\section{References}

1 Andrews, G. and Ball, J. 1982 Asymptotic behavior and change of phase in one dimensional nonlinear viscoelasticity, J. Diff. Eqns, 44,306-341

2 Ball, J. M.

1976 Constitutive equations and existence theorems in nonlinear elastostatics, Heriot-Watt Symp., l, (R. Knops,ed.) Pitman

3 Ball, J. M. 1980 Strict convexity, strong ellipticity, and regularity in the calculus of variations, Math Proc Camb Phil 5oc, $87,501-513$

4 Ball, J. M.

1984 Singular minimizers and their significance in elasticity, Phase Transformations and Material Instabilities in Solids, (Gurtin, M., ed) Academic Press, 1-20

5 Ball, J. M.

6 Ball, J. M. and James, R. energy, to appear Arch. Rat. Mech. Anal.

7 Ball, J. M. and Murat, F. problems for multiple integrals, J. Fnal Anal, 58,(1984),225-253

8 Barrett, C. and Massalski, T. B. 1980 Structure of metals, 3rd edition revised, Pergamon

9 Basinski, Z. S. and Christian, J. W. 1954 Experiments on the martensitic transformations in single crystals of indium-thallium alloys, Acta Met. 2, 149-166

10 Bensoussan, A., Lions, J.-L., and Papanicolaou, G. 1978 Asymptotic analysis for periodic structures

11 Burkart, M. W. and Read, T. A. 1953 Diffusionless phase change in the indium-thallium system, J. of Metals, 5, 1516-1524

12 Chipot, M. and Evans, L. C. 1986 Linearisation at infinity and Lipschitz estimates for certain problems in the calculus of variations, Proc. R. Soc. Edin., 102A, $291-303$

13 Chipot, M., Kinderlehrer, D., and Vergara-Caffarelli, G. 1986 Smoothness of linear laminates, Arch. Rat. Mech. and Anal. 96, 81-96

14 Dacorogna, B.

1982 Weak continuity and weak lower 
semicontinuity of nonlinear functionals, Springer Lecture Notes 922

15 Dacorogna, B. and Fusco, N. 1985 Semi-continuite des fonctionnelles avec contraintes du type "det grad $u>0$ ", Boll. U M I, 6, 179-189

16 Di Perna, R. 1977 Convergence of approximate solutions to conservation laws, Arch. Rat. Mech. Anal., 63, 337-407

17 Eftis, J., MacDonald, D. E., and Arkilic, G. M. 1971 Theoretical calculation of the pressure variation of second-order elastic coefficients for alkali metals, Mater. Sci. Eng. 7, 141-150

18 Eftis, J., MacDonald, D. E., and Arkilic, G. M. 1971 Theoretical calculation of plane wave speeds for alkali metals under pressure, Mater. Sci. Eng. 8, 210-219

19 Ekeland, I. and Temam, R. 1976 Convex analysis and variationial problems, (North-Holland)

20 Ericksen, J. L. 1973 Loading devices and stability of equilibrium, in Nonlinear Elasticity, Academic Press, 161-173

21 Ericksen, J. L. 1977 Special topics in elastostatics, Adv. in appl. mechanics, (C.-5. Yih, ed.) Academic Press 7, 189-243

22 Ericksen, J. L. Arch. Rat. Mech. Anal. 72, 1-13

1979 On the symmetry of deformable crystals,

23 Ericksen, J. L.

Rat. Mech. Anal. 73, 99-124

1980 Some phase transitions in crystals, Arch.

24 Ericksen, J. L.

1981 Some simpler cases of the Gibbs phenomenon for thermoelastic solids, J.of thermal stresses, 4, 13-30

25 Ericksen, J. L. 1981 Changes in symmetry in elastic crystals, IUTAM Symp. Finite Elasticity (Carlson, D.E. and Shield R.T., eds.) M. Nijhoff, 167-177

26 Ericksen, J. L.

1982 Crystal lattices and sublattices, Rend. Sem. Mat. Padova, 68, 1-9

27 Ericksen, J. L. 1983 III posed problems in thermoelasticity theory, Systems of Nonlinear Partial Differential Equations, (Ball, J., ed) D. Reidel, $71-95$

28 Ericksen, J. L. 1984 The Cauchy and Born hypotheses for crystals, Phase Transformations and Material Instabilities in Solids, (Gurtin, M., ed) Academic Press, 61-78

29 Ericksen, J. L. Arch. Rat. Mech. Anal. 88, 337-345

30 Ericksen, J. L. 1986 Stable equilibrium configurations of elastic crystals, Arch. Rat. Mech. Anal. 94, 1-14

31 Ericksen, J. L. 1987 Twinning of crystals 1, Metastability and Incompletely Posed Problems, IMA Vol. Math. Appl. 3,(Antman, S., Ericksen, J.L., Kinderlehrer, D., Müller, l.,eds) Springer, 77-96 
32 Flory, P. J.

1961 Thermodynamic relations for high elastic polymers, Trans. Faraday Soc. 57 (1961), 829-838

33 Fonseca, 1. 1985 Variational methods for elastic crystals, (Thesis ,Univ. of Minn.) Arch. Rat. Mech. Anal.

34 Fonseca, 1.

1987 Stability of elastic crystals (to appear)

35 Fonseca, I. stored energy function of an elastic

The lower quasiconvex envelope of the

36 Fonseca, I. and Tartar, L. crystals

The displacement problem for elastic

37 Hardt, R. and Kinderlehrer, D. 1983 Elastic plastic deformation, Appl. Math. Optim. 10, 203-246

38 James, R. D. 1982 Mechanics of coherent phase transformations in solids, MRL Report, Brown U., Division of Engineering

39 James, R. D. 1984 The arrangement of coherent phases in a loaded body, Phase Transformations and Material Instabilities in Solids, (Gurtin, M.: ed) Academic Press, 79-98

40 James, R. D. Rat. Mech. Anal. 86, 13-37

1984 Stress free joints and polycrystals, Arch.

41 James, R. D.

1986 Phase transformations and non-elliptic free energy, New Perspectives in Thermodynamics (Serrin, J., ed) Springer, 223-239

42 James, R. D. 1986 Displacive phase transformations in solids, J. Mech. Phys. Solids, 34, 359-394

43 James, R. D. 1987 The stability and metastability of quartz, Metastability and Incompletely Posed Problems, IMA Vol. Math. Appl. 3,(Antman, S., Ericksen, J.L., Kinderlehrer, D., Müller, I.,eds) Springer, 147-176

44 Jona, F. and Shirane, $G$.

45 Kelvin and Tait

46 Kinderlehrer, $D$. configurations of crystals, Proc. Symp. Material instabilities in continuum mechanics, Heriot-Watt (Ball, J.M. ed.) Oxford

47 Kinderlehrer, $D$.

1987 Twinning in crystals II, Metastability and Incompletely Posed Problems, IMA Vol. Math. Appl. 3,(Antman, S., Ericksen, J.L., Kinderlehrer, D., Müller, I.,eds) Springer, 185-211

48 Knops, R. J., and Stuart, C. A. 1984 Quasiconvexity and uniqueness of equilibrium solutions in nonlinear elasticity, Arch Rat Mech Anal, 86, 233-249

49 Kohn, R. and Milton, G. 1986 On bounding the effective conductivity of anisotropic composites, Homogenization and effective moduli of materials and media, IMA Volumes in Appl. Math 1 (Ericksen, J. L., Kinderlehrer, D., Kohn, R., and Lions, J.-L.,eds) 97-125

50 Kohn, R. V. and Strang, 6. 1987 Optimal design and relaxation of variational problems, I,CPAM $34,113-137$ 
51 Kohn, R. V. and Strang, G.

1987 Optimal design and relaxation of variational problems, II,CPAM 34, 139-182

52 Kohn, R. V. and Strang, $G$. variational problems, III,CPAM 34, 353-377

1987 Optimal design and relaxation of

53 Lines, M. E. and Glass, A. M. ferroelectrics and related materials, Oxford

54 Love, A. E. H. elasticity, Dover

1948 A treatise on the mathematical theory of

55 Marcellini, $P$.

1986 On the definition and the lower semicontinuity of certain quasiconvex integrals, Ann. Inst. H. Poincarc; Analyse non lineaire, 3, 391-409

56 Mascolo, E. and Schianchi, R. 1983 Existence theorems for non convex problems, J. Math pures et appl, 62, 349-359

57 Meyers, N. G. 1965 Quasiconvexity and lower semi-continuity of multiple variational integrals of any order, Trans. A.M.S. 119, 125-149

58 Milton, G. 1986 Modelling the properties of composites by laminates,Homogenization and effective moduli of materials and media, IMA Volumes in Appl. Math 1 (Ericksen, J. L., Kinderlehrer, D., Kohn, R., and Lions, J.-L.,eds) 150-175

59 Morrey, C. B., Jr. Variations, Springer

1966 Multiple Integrals in the Calculus of

60 Moser, J.

A M S , 120, 286-294

1965 On the volume elements on a manifold, Trans

61 Parry, G. strain, Int. J. Solids Structures, 17, 361-378

62 Pego, R. 1985 Phase transitions: stability and admissibility in one dimensional nonlinear viscoelasticity, IMA preprint 180

63 Pego, R. 1987 Phase transitions in one dimensional nonlinear viscoelasticity: admissibility and stability, Dynamical Problems in Continuum Physics, I M A Vol. Math. Appl. 4 (Bona, J., Dafermos, C., Ericksen, J. L., and Kinderlehrer, D., eds) 277-288

64 Pipkin, A. C. 1986 The relaxed energy density for isotropic elastic membranes, IMA J Appl Math, 36, 85-99

65 Pipkin, A. C. 1986 Some examples of crinkles, Homogenization and effective moduli of materials and media, IMA Volumes in Appl. Math 1 (Ericksen, J. L.s Kinderlehrer, D., Kohn, R., and Lions, J.-L.eds) 182-195

66 Pippard, A. B.

1957 The elements of classical thermodynamics, Cambridge

67 Pitteri, M.

1984 Reconciliation of local and global symmetries of crystals, J. Elasticity 14, 175-190 
crystals, Arch. Rat. Mech. Anal. 88, 25-57

69 Pitteri, M. 1985 On v+ 1 lattices, J. Elasticity, 15, 3-25

70 Pitteri, M. Plasticity, 2, 99-106

1986 On type-2 twins in crystals, int. J.

71 Pitteri, M.

1987 A contribution to the description of natural states for elastic crystalline solids, Metastability and Incompletely Posed Problems, IMA Vol. Math. Appl. 3,(Antman, S., Ericksen, J.L., Kinderlehrer, D., Müller, l.,eds)

Springer, 295-310

72 Simpson, H.C. and Spector, S. J. 1987 on the positivity of the second variation in finite elasticity, Arch. Rat. Mech. Anal. 98, 1-30

73 Slemrod, M. 1986 Interrelationships among mechanics, numerical analysis, compensated compactness, and oscillation theory, Oscillation Theory, Computation, and Methods of Compensated Compactness, 1 M A Vol. Math. Appl. 2, (Dafermos, C., Ericksen, J. L., Kinderlehrer, D., and Slemrod, M. eds) ,309-336.

74 Spencer, L. J. edition, vol VII, 569-591

1911 Crystallography, Encyc. Britannica, 11th

75 Tartar, L. 1979 Compensated compactness and applications to partial differential equations, in Nonlinear analysis and mechanics: Heriot-Watt symp. IV, (Knops, R. J. ed) Pitman, 136-212

76 Temam, R. Gauthier-Villars

1985 Mathematical problems in plasticity,

77 Tisza, L.

1951 On the general theory of phase transformations in solids, (Smoluchowski, R., Mayer, J. E., and Weyl, W. A., eds) Wiley, $1-35$

78 Young, L. C. optimal control theory, W.B. Saunders

1969 Lectures on calculus of variations and

\author{
Département de Mathématiques \\ Université de Metz \\ Ile de Saulcy \\ Metz \\ School of Mathematics \\ University of Minnesota \\ Minneapolis
}

